\title{
Kinematic structure of massive star-forming regions
}

\section{Accretion along filaments ${ }^{\star}$}

\author{
J. Tackenberg ${ }^{1}$, H. Beuther ${ }^{1}$, Th. Henning ${ }^{1}$, H. Linz ${ }^{1}$, T. Sakai ${ }^{2}$, S. E. Ragan ${ }^{1}$, O. Krause ${ }^{1}$, M. Nielbock ${ }^{1}$, \\ M. Hennemann ${ }^{1,3}$, J. Pitann ${ }^{1}$, and A. Schmiedeke ${ }^{1,4}$ \\ 1 Max-Planck-Institut für Astronomie (MPIA), Königstuhl 17, 69117 Heidelberg, Germany \\ e-mail: last-name@mpia.de \\ ${ }^{2}$ Graduate School of Informatics and Engineering, The University of Electro-Communications, Chofu, 182-8585 Tokyo, Japan \\ 3 AIM Paris-Saclay, CEA/DSM/IRFU - CNRS/INSU - Université Paris Diderot, CEA Saclay, 91191 Gif-sur-Yvette Cedex, France \\ ${ }^{4}$ Universität zu Köln, Zülpicher Str. 77, 50937 Köln, Germany
}

Received 22 March 2013 / Accepted 7 January 2014

\begin{abstract}
Context. The mid- and far-infrared view on high-mass star formation, in particular with the results from the Herschel space observatory, has shed light on many aspects of massive star formation. However, these continuum studies lack kinematic information. Aims. We study the kinematics of the molecular gas in high-mass star-forming regions.

Methods. We complemented the PACS and SPIRE far-infrared data of 16 high-mass star-forming regions from the Herschel key project EPoS with $\mathrm{N}_{2} \mathrm{H}^{+}$molecular line data from the MOPRA and Nobeyama $45 \mathrm{~m}$ telescope. Using the full $\mathrm{N}_{2} \mathrm{H}^{+}$hyperfine structure, we produced column density, velocity, and linewidth maps. These were correlated with PACS $70 \mu \mathrm{m}$ images and PACS point sources. In addition, we searched for velocity gradients.

Results. For several regions, the data suggest that the linewidth on the scale of clumps is dominated by outflows or unresolved velocity gradients. IRDC 18454 and G11.11 show two velocity components along several lines of sight. We find that all regions with a diameter larger than $1 \mathrm{pc}$ show either velocity gradients or fragment into independent structures with distinct velocities. The velocity profiles of three regions with a smooth gradient are consistent with gas flows along the filament, suggesting accretion flows onto the densest regions.

Conclusions. We show that the kinematics of several regions have a significant and complex velocity structure. For three filaments, we suggest that gas flows toward the more massive clumps are present.
\end{abstract}

Key words. stars: formation - stars: kinematics and dynamics - ISM: kinematics and dynamics

\section{Introduction}

Despite their rarity, high-mass stars are important for all fields of astronomy. Within the Milky Way they shape and regulate the formation of clusters, influence the chemistry of the interstellar medium, and may even have affected the formation of the solar system (Gritschneder et al. 2012). On larger scales, emission from high-mass stars dominates the emission detected from external galaxies. In addition, massive stars are the origin of heavy elements on all scales. Nevertheless, high-mass star formation is far from being understood (Beuther et al. 2007; Zinnecker \& Yorke 2007).

Sensitive infrared (IR) and (sub-) mm Galactic plane surveys together with results from the Herschel $^{1}$ space observatory (Pilbratt et al. 2010) have shed new light on the cradles of massive stars/clusters and their early formation. Perault et al. (1996) and Egan et al. (1998) discovered extinction patches in the bright mid-IR (MIR) background using the ISO (Kessler et al. 1996) and MSX (Egan et al. 2003) satellites. These extinction patches are similar to the dark patches reported by

\footnotetext{
* Table 3 and Appendix A are available in electronic form at http: //www . aanda.org

1 Herschel is an ESA space observatory with science instruments provided by European-led Principal Investigator consortia and with important participation from NASA.
}

Barnard (1919), which are today known to be connected to low-mass star formation. Soon after this, Carey et al. (1998) established that the so-called infrared dark clouds (IRDCs) are the precursors of high-mass star formation. Today, the Spitzer observatory Galactic plane surveys GLIMPSE at $3.6 \mu \mathrm{m}$, $4.5 \mu \mathrm{m}, 5.8 \mu \mathrm{m}$, and $8 \mu \mathrm{m}$ (Benjamin et al. 2003) and MIPSGAL at $24 \mu \mathrm{m}$ (Carey et al. 2009) allow the systematic search for IRDCs with unprecedented sensitivity (e.g. Peretto \& Fuller 2009).

While Spitzer improved our MIR view of the Galaxy, the Herschel satellite allows observations of the far-IR (FIR). With the PACS (Poglitsch et al. 2010) and SPIRE (Griffin et al. 2010) photometer, high sensitivity and spatial resolution observations between $70 \mu \mathrm{m}$ and $500 \mu \mathrm{m}$ are possible. From correlating data at MIR through submillimeter and millimeter wavelengths, the picture emerged that most star-forming regions are filamentary (André et al. 2010; Men'shchikov et al. 2010; Molinari et al. 2010; Schneider et al. 2010; Hill et al. 2011; Hennemann et al. 2012; Peretto et al. 2012).

In numerical studies, the formation of dense cores and clumps is explained by two scenarios. On the one hand, molecular clouds fragment in a self-similar cascade down to the typical size of dense, quasi-static cores supported by turbulence. These will then form single or multiple gravitationally bound objects (McKee \& Tan 2003; Zinnecker \& Yorke 2007). On the other 
Table 1. Observed IRDCs.

\begin{tabular}{|c|c|c|c|c|c|c|c|c|}
\hline Source & [hh mm ss.s] & $\operatorname{Dec}(\mathrm{J} 2000)$ & Gal. longitude & Gal. latitude & Distance & $\begin{array}{c}\text { Beam } \\
\text { size } \\
{[\mathrm{pc}]}\end{array}$ & $\begin{array}{l}\text { Minimum } \\
\mathrm{N}_{2} \mathrm{H}^{+} \\
\text {detection } \\
\times 10^{12} \mathrm{~cm}^{-2} \\
\end{array}$ & $\begin{array}{c}\text { Minimum } \\
\mathrm{N}_{2} \mathrm{H}^{+} \\
\text {detection } 0.4 \mathrm{~km} \mathrm{~s}^{-1} \\
\times 10^{12} \mathrm{~cm}^{-2}\end{array}$ \\
\hline \multicolumn{9}{|c|}{ Observed with Nobeyama $45 \mathrm{~m}$} \\
\hline IRDC 18223 & 182510.7 & -124512 & 18.613 & -0.081 & 3.5 & 0.3 & 0.8 & \\
\hline IRDC 18310 & 183344.7 & -082236 & 23.467 & 0.085 & 4.9 & 0.4 & 3.9 & \\
\hline IRDC 18385 & 184117.1 & -050915 & 27.189 & -0.098 & 3.1 & 0.3 & 1.2 & \\
\hline IRDC 18454 & 184758.1 & -015441 & 30.835 & -0.100 & 5.3 & 0.5 & 4.1 & \\
\hline ISOSS J20153 & 201521.4 & +345352 & 72.953 & -0.027 & 1.2 & 0.1 & 8.1 & \\
\hline IRDC 13.90 & 181726.1 & -170526 & 13.906 & -0.473 & 2.6 & 0.2 & 1.4 & \\
\hline \multicolumn{9}{|c|}{ Observed with Mopra } \\
\hline IRDC 18102 & 181312.2 & -175934 & 12.632 & -0.016 & 2.6 & 0.6 & 5.7 & 3.3 \\
\hline IRDC 18151 & 181755.3 & -120729 & 18.335 & 1.778 & 2.7 & 0.6 & 3.9 & 2.8 \\
\hline IRDC 18182 & 182112.2 & -143246 & 16.577 & -0.069 & 3.4 & 0.7 & 3.2 & 2.2 \\
\hline IRDC 18306 & 183329.9 & -083207 & 23.298 & 0.066 & 3.6 & 0.8 & 1.5 & 1.6 \\
\hline IRDC 18308 & 183333.1 & -083746 & 23.220 & 0.011 & 4.4 & 1.0 & 2.9 & 2.1 \\
\hline IRDC 18337 & 183629.6 & -074033 & 24.402 & -0.197 & 3.7 & 0.8 & 1.8 & 1.1 \\
\hline IRDC 19.30 & 182558.1 & -120509 & 19.293 & 0.060 & 2.4 & 0.5 & 7.6 & 1.3 \\
\hline IRDC 11.11 & 181020.0 & -192505 & 11.056 & -0.107 & 3.4 & 0.7 & 2.4 & 1.6 \\
\hline IRDC 15.05 & 181740.4 & -154912 & 15.052 & 0.080 & 3.0 & 0.7 & 3.4 & 1.4 \\
\hline IRDC 28.34 & 184248.1 & -040151 & 28.361 & 0.080 & 4.5 & 1.0 & 4.5 & 3.2 \\
\hline IRDC 48.66 & 192140.7 & +135032 & 48.666 & -0.263 & 2.6 & 0.6 & 2.3 & 0.8 \\
\hline
\end{tabular}

Notes. Position Cols. (2-5) give the center coordinate of the maps. The actual areas that were mapped are displayed in Fig. 1 through Fig. 4. The distances are adopted from (Ragan et al. 2012a) with references therein. The detected $\mathrm{N}_{2} \mathrm{H}^{+}$minimum is the minimum plotted in Figs. 1 through 4. For sources observed with MOPRA we also give the improved minimum detection when smoothing the spectra to a velocity resolution of $0.4 \mathrm{~km} \mathrm{~s}^{-1}$. Also observed with the NRO 45m telescope, but not detected, were IRDC20081, ISOSSJ19357, ISOSSJ19557, and ISOSSJ20093.

hand, in the dynamical theory molecular clouds are formed from large-scale flows of atomic gas as transient objects (Mac Low \& Klessen 2004; Klessen et al. 2005; Heitsch \& Hartmann 2008; Clark et al. 2012). Within these transient structures, supersonic turbulence compresses some fraction of the gas to filaments, clumps, and dense cores. If gravity dominates, the cores collapse. In contrast to the quasi-static cores, these cores constantly grow in mass. When, by chance, some cores accrete mass faster than others due to their higher initial gravitational potential, this is called competitive accretion (Bonnell et al. 2004). Also dynamical, but of reversed reasoning, in the fragmentation-induced starvation scenario by Peters et al. (2010), individual massive dense cores build from the large-scale flows, and an accompanying cluster of smaller cores drag away material from the main core and diminish its mass accretion.

The Earliest Phases of Star formation (EPoS, PI O. Krause) is a guaranteed time Herschel key program for investigating 14 low-mass and 45 high-mass star-forming regions. The lowmass observations have been summarized in Launhardt et al. (2013), and the high-mass part has been described in Ragan et al. (2012a). The high-mass part of the project provides an excellent target list for studying the kinematics in star-forming regions. This is the ultimate goal of this paper, using $\mathrm{N}_{2} \mathrm{H}^{+}$molecular line data.

\section{Observations and analysis}

\subsection{EPoS - A Herschel key project}

All 45 high-mass EPoS sources were observed with the Herschel satellite (Pilbratt et al. 2010) at $70 \mu \mathrm{m}, 100 \mu \mathrm{m}, 160 \mu \mathrm{m}, 250 \mu \mathrm{m}$, $350 \mu \mathrm{m}$, and $500 \mu \mathrm{m}$ with a spatial resolution of $5.6^{\prime \prime}, 6.8^{\prime \prime}$, 11.3", 18.1", 25.2", and 36.6", respectively (Poglitsch et al. 2010; Griffin et al. 2010). The observations were performed in two orthogonal directions and the data reduction has been performed using HIPE (Ott 2010) and scanamorphos (Roussel 2013). A more detailed description of the data reduction is given in Ragan et al. (2012a).

Out of the 45 Herschel EPoS high-mass sources we selected a subsample of 17 regions given in Table 1 that cover each important evolutionary stage: promising high-mass starless core candidates, IRDCs with weak MIR and FIR sources, indicative of early ongoing star formation activity, and known high-mass protostellar objects (HMPOs).

The protostellar core population was previously characterized in Ragan et al. (2012a) using Herschel photometry, supplemented by Spitzer, IRAS, and MSX data. By modeling the spectral energy distributions (SEDs), the authors fit the temperature, luminosity, and mass of each protostellar core in the sample.

\subsection{Nobeyama $45 \mathrm{~m}$ observations}

Between April 7 and 122010 the BEam Array Receiver System (BEARS, Sunada et al. 2000) on the Nobeyama Radio Observatory $\left(\mathrm{NRO}^{2}\right) 45 \mathrm{~m}$ telescope was used to map six of the regions in $\mathrm{N}_{2} \mathrm{H}^{+}$; the details are given in Table 1. At a frequency of the $\mathrm{N}_{2} \mathrm{H}^{+}(1-0)$ transition of $93.173 \mathrm{GHz}$, the spatial resolution of the NRO $45 \mathrm{~m}$ telescope is $18^{\prime \prime}$ (HPBW) and the observing mode with a bandwidth of $32 \mathrm{MHz}$ has a frequency resolution of $62.5 \mathrm{kHz}$, or $0.2 \mathrm{~km} \mathrm{~s}^{-1}$. All observations were performed using on-the-fly (OTF) mapping in varying weather conditions, with an average system temperature of $280 \mathrm{~K}$ and high precipitable water vapors between $3 \mathrm{~mm}$ and $9 \mathrm{~mm}$. The pointing was made using the single-pixel receiver $\mathrm{S} 40$ tuned to $\mathrm{SiO}$. As

2 Nobeyama Radio Observatory is a branch of the National Astronomical Observatory of Japan, National Institutes of Natural Sciences. 
Table 2. Summary of the different dust data and the corresponding properties.

\begin{tabular}{lcccccc}
\hline \hline Dust data & $\begin{array}{c}\text { Wavelength } \\
{[\mu \mathrm{m}]}\end{array}$ & $\begin{array}{c}\text { Beam FWHM } \\
{\left[{ }^{\prime \prime}\right]}\end{array}$ & $\begin{array}{c}\text { rms noise } \\
{\left[\mathrm{mJy} \mathrm{beam}^{-1}\right]}\end{array}$ & $\begin{array}{c}\kappa_{\text {dust }} \\
{\left[\mathrm{cm}^{2} \mathrm{~g}^{-1}\right]}\end{array}$ & $\begin{array}{c}\text { Lowest contour } \\
{\left[\mathrm{mJy} \mathrm{beam}^{-1}\right]}\end{array}$ & $\begin{array}{c}\text { Column density threshold } \\
{\left[\mathrm{cm}^{-2}\right]}\end{array}$ \\
\hline ATLASGAL/APEX & 870 & 19.2 & 50 & 1.42 & 310 & $1 \times 10^{22}$ \\
MAMBO/IRAM 30 m & 1200 & 10.5 & 17 & 0.79 & 60 & $2 \times 10^{22}$ \\
SCUBA/JCMT & 850 & 14.0 & 14 & 1.48 & 176 & $1 \times 10^{22}$ \\
\hline
\end{tabular}

Notes. The column densities were calculated under the assumptions given in Sect. 2.6 for $20 \mathrm{~K}$. The last column is the column density corresponding to the lowest emission contour used within CLUMPFIND.

pointing sources we used the $\mathrm{SiO}$ masers of the late-type stars V468 Cyg, IRC+00363, and R Aql. Although the wind conditions contributed to the pointing uncertainties, the pointing is better than a third of the beam.

For the data reduction we used NOSTAR (Sawada et al. 2008), a software package provided by the NRO for OTF data. The data were sampled to a spatial grid of $7.5^{\prime \prime}$ and smoothed to a spectral resolution of $0.5 \mathrm{~km} \mathrm{~s}^{-1}$. To account for the different efficiencies of the 25 receivers in the BEARS array we corrected each pixel to the efficiency of the $S 100$ receiver, using individual correction factors and a beam efficiency of $\eta=0.46$ to calculate main-beam temperatures. The noisy edges due to less coverage were removed within NOSTAR by suppressing pixels in the final maps with an rms noise above $0.15 \mathrm{~K}$. The resulting antenna temperature maps have an average rms noise between $0.12 \mathrm{~K}$ and $0.13 \mathrm{~K}$ per beam.

\subsection{MOPRA observations}

The 11 sources, listed in Table 1 were mapped with the $22 \mathrm{~m}$ MOPRA radio telescope, operated by the Australia Telescope National Facility (ATNF) in OTF mode. The observations were carried out in 2010, June 1 to 5 and 25 to 27 , as well as July 7 through 9. High precipitable water vapors during the observations result in system temperatures mostly of between $200 \mathrm{~K}$ and $300 \mathrm{~K}$. Observations with system temperatures higher than $500 \mathrm{~K}$ were ignored during the data reduction.

We employed 13 of the MOPRA spectrometer (MOPS) zoom bands, each of $138 \mathrm{MHz}$ width and 4096 channels, resulting in a velocity resolution of $0.11 \mathrm{~km} \mathrm{~s}^{-1}$ at $90 \mathrm{GHz}$. The spectral setup covered transitions of $\mathrm{CH}_{3} \mathrm{CCH}, \mathrm{H}^{13} \mathrm{CN}, \mathrm{H}^{13} \mathrm{CO}^{+}$, $\mathrm{SiO}, \mathrm{C}_{2} \mathrm{H}, \mathrm{HNCO}, \mathrm{HCN}, \mathrm{HCO}^{+}, \mathrm{HNC}, \mathrm{HCCCN}, \mathrm{CH}_{3} \mathrm{CN},{ }^{13} \mathrm{CS}$, and $\mathrm{N}_{2} \mathrm{H}^{+}$in the $90 \mathrm{GHz}$ regime (for details on the transitions and their excitation conditions see Vasyunina et al. 2011). At this wavelength, the MOPRA beam FWHM is 35.5" and the beam efficiency is assumed to be constant over the frequency range with $\eta=0.49$ (Ladd et al. 2005). The data were reduced using LIVEDATA and GRIDZILLA, an mapping analysis package provided by the ATNF. To improve the signal-to-noise ratio we spatially smoothed the data to a beam FWHM of $46^{\prime \prime}$ within (and as suggested by) gridzilla. The final maps were smoothed to a spectral resolution of $0.21 \mathrm{~km} \mathrm{~s}^{-1}-0.23 \mathrm{~km} \mathrm{~s}^{-1}$ (depending on the transition frequency). Spectra with an $\mathrm{rms}$ noise above $0.12 \mathrm{~K}$ have been removed; this affected pixels at the edges. The resulting average rms noise of the individual maps is then below $0.09 \mathrm{~K} /$ beam.

The observed regions of interest are dense but still cold. Therefore, with the achieved sensitivity at the given spatial resolution we did not detect the more complex or lowabundance molecules. For example, although $\mathrm{SiO}(2-1)$ has been detected toward several positions we mapped (Sridharan et al. 2002; Sakai et al. 2010), the strongest SiO emitter found by Linz et al. (in prep., G28.34-2) is at our noise level and therefore not detected. Commonly detected and reasonably well mapped are $\mathrm{HCN}(1-0), \mathrm{HNC}(1-0), \mathrm{HCO}^{+}(1-0), \mathrm{H}^{13} \mathrm{CO}^{+}$ (1-0), and $\mathrm{N}_{2} \mathrm{H}^{+}(1-0)$. As we discuss in Sect. 2.5, we concentrate on $\mathrm{N}_{2} \mathrm{H}^{+}$, a well-known cold dense gas tracer.

\subsection{Dust continuum}

To trace the total cold gas we used the cold dust emission as a tracer of the molecular gas. Because most of the selected sources lie within the Galactic plane, the APEX $870 \mu \mathrm{m}$ survey ATLASGAL (Schuller et al. 2009; Contreras et al. 2013) covers all but two sources. Its beam size is $19.2^{\prime \prime}$ and its average $\mathrm{rms}$ noise is $50 \mathrm{mJy} /$ beam. IRDC 18151 with a Galactic latitude of $\sim 1.7^{\circ}$ is not covered by ATLASGAL. We used IRAM $30 \mathrm{~m}$ MAMBO data instead (Beuther et al. 2002b). At a wavelength of $1.2 \mathrm{~mm}$, the beam width is $10.5^{\prime \prime}$, and the rms noise in the dust map is $17 \mathrm{mJy} /$ beam. In addition, ISOSS J20153 is outside the coverage of ATLASGAL. Here we used $850 \mu \mathrm{m}$ data from the SCUBA camera at the $\mathrm{JCMT}^{3}$ (Hennemann et al. 2008). The beam width is $14^{\prime \prime}$ and the rms noise $14 \mathrm{mJy} /$ beam. A summary of the properties of the submm data is given in Table 2 .

\section{5. $\mathrm{N}_{2} \mathrm{H}^{+}$hyperfine fitting}

Our molecular line study focuses on the $\mathrm{N}_{2} \mathrm{H}^{+} J=1-0$ line as dense molecular gas tracer. Using the Einstein A and collison coefficient for a temperature of $20 \mathrm{~K}$ (Schöier et al. 2005), its critical density is $1.6 \times 10^{5} \mathrm{~cm}^{-3}$, and its hyperfine structure allows one to reliably measure its optical depth and thus the distribution over a wide range of densities. In addition, the velocity and linewidth can be measured without being affected by optical depth effects. Finally, it is detected toward both low- and high-mass star-forming regions of various evolutionary stages (Schlingman et al. 2011). Therefore, it is well-suited for studies of young high-mass star-forming regions.

To extract the $\mathrm{N}_{2} \mathrm{H}^{+}$line parameters, we fit a $\mathrm{N}_{2} \mathrm{H}^{+}$hyperfine structure to each pixel using class from the GILDAS $S^{4}$ package. For every spectrum we calculated the rms and the peak intensity of the brightest component derived from the fit parameters. When the peak was higher than three times the rms value, the fit parameters peak velocity and the linewidth together with an integrated intensity were stored to a parameter map. Otherwise, the pixel was left blank. The low detection threshold of $3 \sigma$ is justified for two reasons. (1) For only a very limited number of pixels that fulfill the $3 \sigma$ criterion, the fitted linewidth is twice the

3 The James Clerk Maxwell Telescope is operated by the Joint Astronomy Centre on behalf of the Particle Physics and Astronomy Research Council of the United Kingdom, the Netherlands Association for Scientific Research, and the National Research Council of Canada. 4 http://www . iram. fr/IRAMFR/GILDAS 
channel width or smaller. Therefore, introducing an additional check on the integrated intensity, such as a $5 \sigma$, does not improve the fit reliability. (2) The resulting parameter maps only show a smooth transition in each parameter relative to the neighboring pixels. For the same pixels, smoothing over a larger area would increase the signal-to-noise ratio but would worsen the resolution. Therefore, the small-scale structure would be lost. For our purposes, fitting the hyperfine structure even in low signal-tonoise ratio maps provides reliable results.

From the integrated intensity $\left(\int T_{\mathrm{mb}}\right)$, determined as the sum over channels times the channel width, and the fitted optical depth $\tau$, we calculated the column densities of $\mathrm{N}_{2} \mathrm{H}^{+}$. We used the formula from Tielens (2005),

$$
\begin{aligned}
N_{J} & =1.94 \times 10^{3} \frac{v^{2} \int T_{\mathrm{mb}}}{A_{u}} \times \frac{\tau}{1-\mathrm{e}^{\tau}}(\text { for } J+1 \text { to } J) \\
N_{\text {tot }} & =\frac{Q}{g_{\mathrm{u}}} \times N_{J} \times \mathrm{e}^{E_{u} / k T_{\mathrm{ex}}},
\end{aligned}
$$

where $N_{\mathrm{J}}$ is the number of molecules in the $J$ th level, and $N_{\text {tot }}$ the total number of molecules. $A_{\mathrm{u}}$ is the Einstein A coefficient of the upper level, $E_{\mathrm{u}} / \mathrm{k}$ is the excitation energy of the upper level in $\mathrm{K}$, both adopted from Schöier et al. (2005) and Vasyunina et al. (2011); Q is the partition function of the given level, taken from the Cologne Database for Molecular Spectroscopy (Müller et al. 2005), and $g_{\mathrm{u}}$ is the degeneracy of the energy level.

The rms of our observations limits the detection of signal, but the $\mathrm{N}_{2} \mathrm{H}^{+}$column density also depends on the measured opacity and assumed temperature. As we discuss in Sect. 3.1, we used a constant gas temperature of $20 \mathrm{~K}$. Varying the temperature by up to $5 \mathrm{~K}$, the calculated column densities vary by less than $25 \%$. Taking the error on the integrated intensity and the partition function into account as well, we assumed the error on the column density to be on the order of $50 \%$. With the given rms toward the edge of the MOPRA data, our theoretical $5 \sigma \mathrm{N}_{2} \mathrm{H}^{+}$detection limit in the optically thin case is given by $1.5 \times 10^{12} \mathrm{~cm}^{-2}$ for the velocity resolution of $0.2 \mathrm{~km} \mathrm{~s}^{-1}$. Since most sources have considerable optical depth, the lowest measured $\mathrm{N}_{2} \mathrm{H}^{+}$column density is higher. The lowest calculated values are given in Table 1. Similarly, for the Nobeyama $45 \mathrm{~m}$ data the $5 \sigma$ detection limit is $2.1 \times 10^{12} \mathrm{~cm}^{-2}$. Since some central regions for instance of IRDC 18223 have a much lower rms of only $0.05 \mathrm{~K}$ instead of $0.12 \mathrm{~K}$, here the lowest calculated values are even lower.

The uncertainties on the fit parameters velocity and linewidth are mainly constrained by the signal-to-noise ratio and the line shape. Since even the broadest velocity resolution within our sample of $0.5 \mathrm{~km} \mathrm{~s}^{-1}$ resolves the lines, the uncertainties on the linewidth are similar for all data. Spectra with a signal-to-noise ratio better than $7 \sigma$ and Gaussian-shaped line profiles have typical linewidth uncertainties of below $5 \%$, while non-Gaussian line profiles and low signal-to-noise ratios may lead to uncertainties of up to $20 \%$. Instead, the recovery of the peak velocity shows an additional slight velocity resolution dependency. Still, both line shape and signal-to-noise ratio dominate, and down to a peak line strength of $3 \sigma$, the uncertainties on the velocity are below $0.2 \mathrm{~km} \mathrm{~s}^{-1}$.

\subsection{Identification of dust peaks}

To set the molecular line data in context to its environment we used the dust continuum to obtain gas column densities and masses. For the calculation of column densities from

\section{fluxes we used}

$N_{\text {gas }}=\frac{R F_{\lambda}}{B_{\lambda}(\lambda, T) \mu m_{\mathrm{H}} \kappa \Omega}$,

with the gas-to-dust mass ratio $R=100, F_{\lambda}$ the flux at the given wavelength, $B_{\lambda}(\lambda, T)$ the blackbody radiation as a function of wavelength and temperature, $\mu$ the mean molecular weight of the ISM of 2.8, $m_{\mathrm{H}}$ the mass of a hydrogen atom, and the beam size $\Omega$. Assuming typical beam-averaged volume densities in the dense gas of $10^{5} \mathrm{~cm}^{-3}$ and dust grains with thin ice mantles, we interpolated the dust mass absorption coefficient from Ossenkopf \& Henning (1994) to the desired wavelength. The corresponding dust opacities for the different wavelengths are listed in Table 2.

The gas and dust temperatures should be coupled at densities typical for dense clump $\left(>10^{5} \mathrm{~cm}^{-3}\right.$, Goldsmith 2001), and have been measured to be between $15 \mathrm{~K}$ and $20 \mathrm{~K}$ (Sridharan et al. 2005; Pillai et al. 2006; Peretto \& Fuller 2010; Battersby et al. 2011; Wilcock et al. 2011; Vasyunina et al. 2011; Wienen et al. 2012; Wilcock et al. 2012). Since most regions in our survey already show signs of ongoing star formation, we assumed a single temperature value of $20 \mathrm{~K}$ for all clumps.

With the distance $\mathrm{d}$ as additional parameter, the mass can be calculated from the integrated flux in a similar way as given above,

$M_{\mathrm{gas}}=\frac{R \mathrm{~d}^{2} F_{\lambda}}{B_{\lambda}(\lambda, T) K}$.

To identify emission peaks and their connected fluxes in the dust maps we used CLUMPFIND (Williams et al. 1994). Since we aim to compare our results to the dense gas measured by $\mathrm{N}_{2} \mathrm{H}^{+}$, we selected a lowest emission contour corresponding to $1 \times 10^{22} \mathrm{~cm}^{-2}$ ( $>6 \sigma$ for ATLASGAL, $>12 \sigma$ for SCUBA), or, in the case of ISOSS J20153, $2 \times 10^{22} \mathrm{~cm}^{-2}(>3 \sigma)$. Additional levels were added in steps of $3 \sigma$, see Table 2 . All clumps for which we mapped the peak position are listed together with their column density and mass in Table 3 . For clumps that have common names in the literature, we adopted their previous labeling. The clump names and references are given in Table 3 as well.

The uncertainties on both the gas column density and mass are dominated by the dust properties. The flux calibration of the ATLASGAL data is reliable within $15 \%$, and typical peak and clump-integrated fluxes are an order of magnitude higher than the rms of the data. The uncertainties on the dust properties are difficult to assess, but from comparison with other values (e.g. Hildebrand 1983) or using slightly different parameters within the same model (Ossenkopf \& Henning 1994), we assumed them to be on the order of a factor two. Together with the uncertainties from the dust temperature, we estimate the total uncertainties on the column densities to be a factor of $\sim 3$. For the gas mass, the uncertainty of the distance of $\sim 0.5 \mathrm{kpc}$ introduces an additional error of $\sim 50 \%$. Therefore, we estimate the total uncertainty of the gas mass to be on the order of a factor of five.

\subsection{Abundance ratio}

For positions where not only dust continuum but also $\mathrm{N}_{2} \mathrm{H}^{+}$has been detected we calculated the abundance ratios. With a resolution of $18^{\prime \prime}$ and $19.2^{\prime \prime}$, the Nobeyama $45 \mathrm{~m}$ data have almost the same resolution as the ATLASGAL $870 \mu \mathrm{m}$ data. Therefore we calculated the $\mathrm{N}_{2} \mathrm{H}^{+}$abundance ratio by plain division, taking the different beam size into account, but did not smooth the data. To calculate abundance ratios for sources observed with 
MOPRA at a resolution of 46", we applied a Gaussian smoothing to the dust data to have both at a common resolution. We then calculated the $\mathrm{N}_{2} \mathrm{H}^{+}$abundance for the $46^{\prime \prime}$ beam. For the analysis, we only considered dust measurements in the Gaussiansmoothed images above $3 \sigma$.

\section{Morphology of the dense gas}

In the following section we concentrate on the $\mathrm{N}_{2} \mathrm{H}^{+}$observations. First we compare the $\mathrm{N}_{2} \mathrm{H}^{+}$with the cold dust distribution as measured by ATLASGAL and set it in context with the PACS $70 \mu \mathrm{m}$ measurements. Then we describe the velocity and linewidth distribution of the dense gas.

\subsection{Comparing integrated $\mathrm{N}_{2} \mathrm{H}^{+}$and dust continuum emission}

The left panels of Figs. 1 through 4 display the PACS $70 \mu \mathrm{m}$ maps with the long-wavelength dust continuum contours on top, the second-left panel is the $\mathrm{N}_{2} \mathrm{H}^{+}$column density. They clearly show that the $\mathrm{N}_{2} \mathrm{H}^{+}$detection and column density agrees in general with the measured dense gas emission, almost independent of the evolutionary state of the clump.

The southern component of IRDC G11.11 is peculiar, see Fig. 2. While for the northern component the molecular gas traced by $\mathrm{N}_{2} \mathrm{H}^{+}$agrees quite well with the cold gas traced by thermal dust emission, the two dense gas tracers disagree for the southern part. Comparing the brightest peak in the ATLASGAL data with the column density peak of the $\mathrm{N}_{2} \mathrm{H}^{+}$emission, we find a positional difference of $37^{\prime \prime}$, which is on the order of the beam size. Since the northern and southern component have been observed independently, a pointing error might explain the offset. However, before and in between the OTF observations we checked the pointing and the offset is considerably larger than the anticipated pointing uncertainty. Therefore, we cannot explain the spatial offset of the southern map well.

For IRDC 18182, the bright northwestern component is connected to IRAS $18182-1433$ at a velocity of $59.1 \mathrm{~km} \mathrm{~s}^{-1}$ (Bronfman et al. 1996) and a distance of $4.5 \mathrm{kpc}$ (Faúndez et al. 2004). Instead, the region of interest is the IRDC in the southeast at a distance of $3.44 \mathrm{kpc}$ with a velocity of $41 \mathrm{~km} \mathrm{~s}^{-1}$ (Beuther et al. 2002a; Sridharan et al. 2005).

IRDC 18308 has been selected within this sample for its infrared dark cloud north of the HMPO IRAS 18308-0841. At its distance of $4.4 \mathrm{kpc}$ we did not detect the $\mathrm{N}_{2} \mathrm{H}^{+}$emission from the IRDC with the velocity resolution of $0.2 \mathrm{~km} \mathrm{~s}^{-1}$. To overcome the sensitivity issue we smoothed the velocity resolution to $0.4 \mathrm{~km} \mathrm{~s}^{-1}$ and were then able to trace the dense gas of the IRDC within IRDC 18308 . For IRDC 18306 the situation is similar, we traced the HMPO, but not the IRDC. Unfortunately, even with a velocity resolution of only $0.4 \mathrm{~km} \mathrm{~s}^{-1}$ we were unable to detect $\mathrm{N}_{2} \mathrm{H}^{+}$from the IRDC. Therefore, we excluded IRDC 18306 from the discussion and show its dense gas properties in Appendix A.2. To offer a better picture of the different regions we display in Figs. 1 through 4 the results from the smoothed maps, where helpful. Because the coverage for many clumps is sufficient in the higher resolution data, we used the $0.2 \mathrm{~km} \mathrm{~s}^{-1}$ data for our analysis.

The total gas peak column densities over the 19.2" APEX $870 \mu \mathrm{m}$ beam as given in Table 3 range from $1.4 \times 10^{22} \mathrm{~cm}^{-2}$ to $8 \times 10^{23} \mathrm{~cm}^{-2}$, and the median averaged peak column density of clumps that have been mapped is $2.6 \times 10^{22} \mathrm{~cm}^{-2}$. When we consider only clumps for which the peak position has a detected $\mathrm{N}_{2} \mathrm{H}^{+}$signal, the median averaged peak column density becomes $3.0 \times 10^{22} \mathrm{~cm}^{-2}$. For the lower limit one needs to keep in mind that we require a minimum column density threshold of $1.0 \times 10^{22} \mathrm{~cm}^{-2}$ for a clump to be detected. The upper limit is set by IRDC 18454-mm1 (adopted from W43mm1, Motte et al. 2003; Beuther et al. 2012), the brightest clump within IRDC 18454 and a well-known site of massive star formation. All other clumps with peak column densities higher than $1 \times 10^{23} \mathrm{~cm}^{-2}$ (IRDC 18151-1, IRDC 18182-1, and G 28.34-2) host evolved cores and could be warmer than $20 \mathrm{~K}$. Nevertheless, to calculate the column densities we assumed a constant average dense gas temperature of $20 \mathrm{~K}$. While this is appropriate for most IRDCs in this sample with ongoing early star formation (cf. point sources in Ragan et al. 2012a), using a higher temperature would decrease their peak column densities. With the exception of W43-mm1, the upper limit of column densities found within our survey's sources then becomes $\sim 1 \times 10^{23} \mathrm{~cm}^{-2}$ on scales of the beam size.

\section{2. $\mathrm{N}_{2} \mathrm{H}^{+}$abundance}

To study the details of the correlation between the dense gas and the related $\mathrm{N}_{2} \mathrm{H}^{+}$column density, Fig. 5 shows the pixel-by-pixel correlation between the $\mathrm{N}_{2} \mathrm{H}^{+}$abundance ratio versus the flux ratio between the Herschel $160 \mu \mathrm{m}$ and $250 \mu \mathrm{m}$ bands. One should keep in mind that as explained in Sect. 2.7, the abundance ratio refers to different beam sizes, depending on the telescope that was used for the observations. The flux ratio of the two PACS bands, or color temperature, can be considered as a proxy of the dust temperature. For higher temperatures, the peak of the SED moves to shorter wavelengths, the $160 \mu \mathrm{m}$ becomes stronger relative to the $250 \mu \mathrm{m}$ flux. Therefore, higher temperatures have higher FIR flux ratios. To derive proper temperatures, a pixel by pixel SED fitting is required, which will be done in an independent paper (Ragan et al., in prep.). A known problem in the context of Herschel data are the unknown background flux levels. Since we only discuss trends within individual regions, we can safely neglect this problem. Thus, the flux ratios between the different regions are not directly comparable.

To allow a comparison between IRDCs and regions that do not show up in extinction, we marked pixels that lie within regions of high extinction in green. These regions were selected by visually identifying the $70 \mu \mathrm{m}$ flux levels below which extinction features are observed. Because the $70 \mu \mathrm{m}$ dark regions have no sharp boundaries, the chosen levels are not unambiguous.

Figure 5 shows no strong overall correlation between the $\mathrm{N}_{2} \mathrm{H}^{+}$abundance and the flux ratio. On the one hand, IRDC 18223 is an example where there seems to be a correlation between the temperature and the $\mathrm{N}_{2} \mathrm{H}^{+}$abundance. The highest abundance ratios are found at FIR flux ratios of the bulge of the pixel distribution, while toward higher temperatures the abundance seems to decrease systematically. On the other hand, in G11.11 the $\mathrm{N}_{2} \mathrm{H}^{+}$abundance varies over two orders of magnitude, but shows no correlation with the FIR flux ratio. For example, in G11.11 and G28.34, there seems to be a trend that the $\mathrm{N}_{2} \mathrm{H}^{+}$abundances become even larger toward the edges of our $\mathrm{N}_{2} \mathrm{H}^{+}$mapping. However, in these regions we are limited by the sensitivity of our dust measurements.

Marked by an $\times$ in Fig. 5 are the pixels containing PACS point sources. In addition, we distinguished between sources that have only been detected at $70 \mu \mathrm{m}$ and longwards, MIPS-dark sources, and PACS sources with a $24 \mu \mathrm{m}$ counterpart, so-called MIPS-bright sources. When the $24 \mu \mathrm{m}$ image was saturated at the given position, sources were considered as MIPS-bright as 

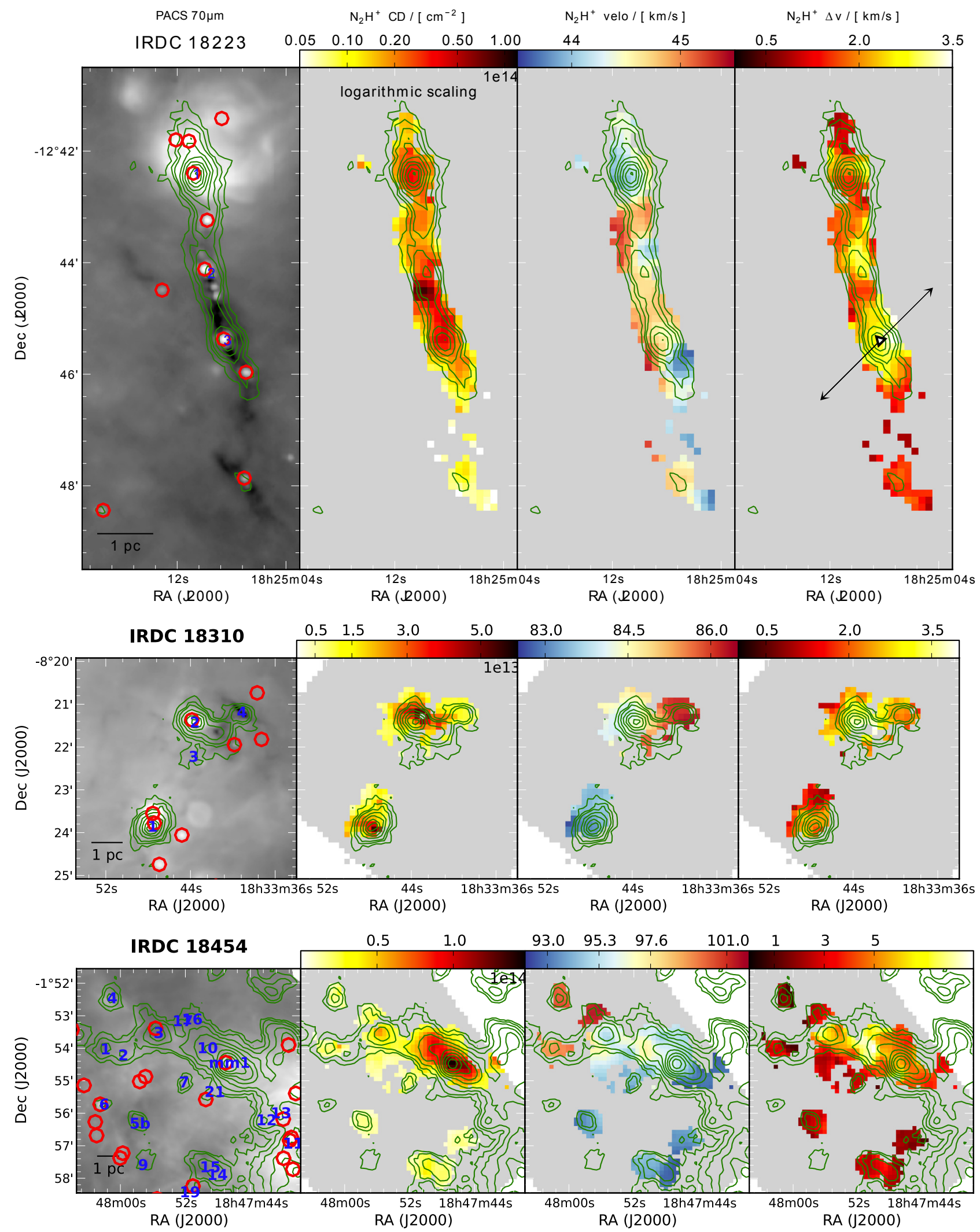

Fig. 1. Parameter maps of the regions IRDC 18223, IRDC 18310, and IRDC 18454 mapped with the Nobeyama $45 \mathrm{~m}$ telescope, in top, middle, and bottom panel, respectively. The left panels of each row are the PACS $70 \mu \mathrm{m}$ maps with the PACS point sources detected by Ragan et al. (2012a) indicated by red circles, the blue numbers refer to the submm continuum peaks as given in Table 3 . The second panels display the $\mathrm{N}_{2} \mathrm{H}^{+}$column density derived from fitting the full $\mathrm{N}_{2} \mathrm{H}^{+}$hyperfine structure. The third and fourth panels show the corresponding velocity and linewidth (FWHM) of each fit. For IRDC 18223, and IRDC 18310 the contours from ATLASGAL $870 \mu \mathrm{m}$ are plotted with the lowest level representing 0.31 Jy, and continue in steps of $0.3 \mathrm{Jy}$. The contour levels for IRDC 18454 are logarithmically spaced, with 10 levels between $0.31 \mathrm{Jy}$ and $31 \mathrm{Jy}$. The column density scale of IRDC 18223 is logarithmic. The arrow in the fourth panel of IRDC 18223 is taken from Fig. 4 of Fallscheer et al. (2009), indicating the outflow direction. 

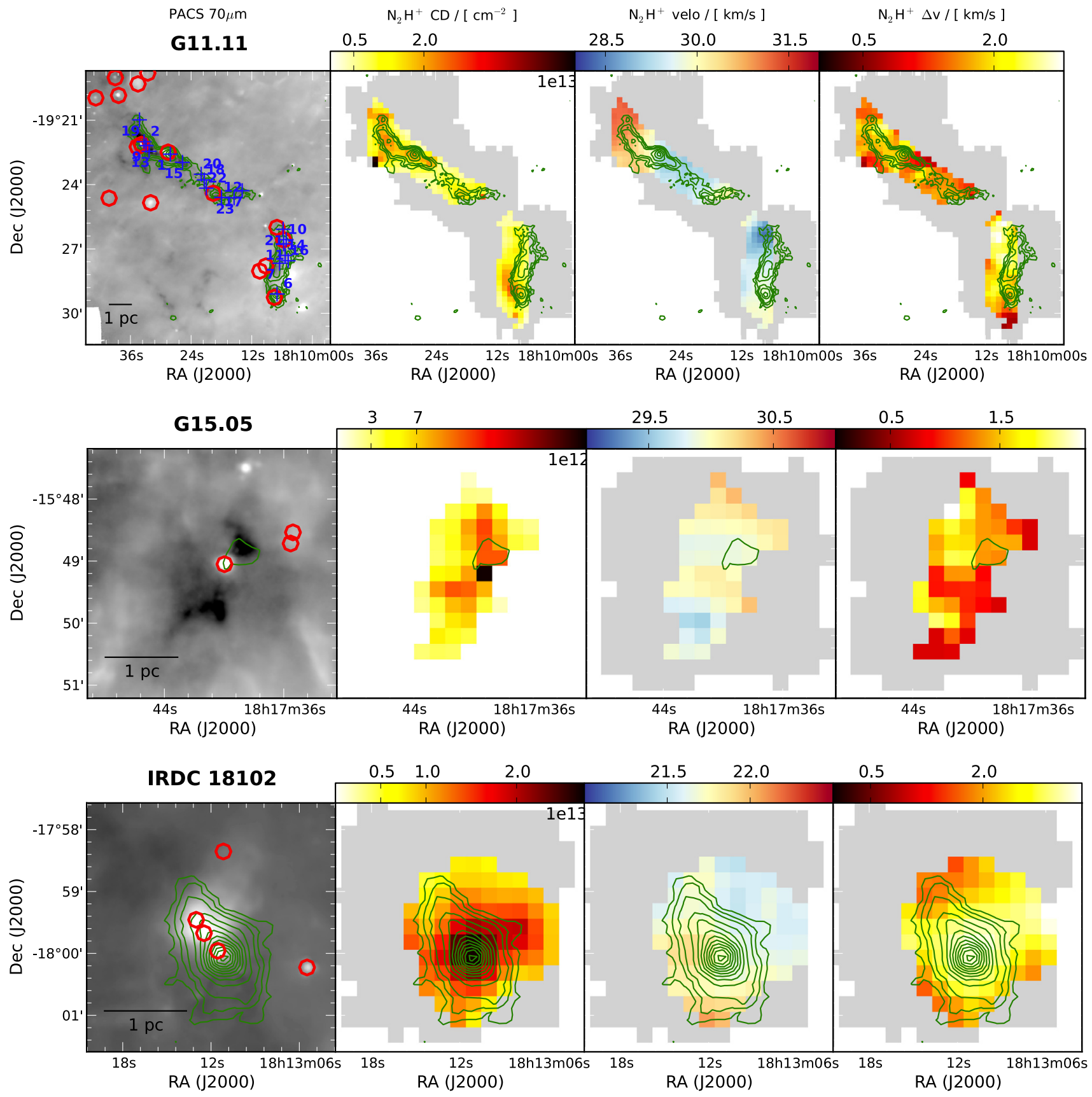

Fig. 2. Parameter maps of the regions G11.11, G15.05, and IRDC 18102, mapped with the MOPRA telescope. The left panels of each row are the PACS $70 \mu \mathrm{m}$ maps with the PACS point sources detected by Ragan et al. (2012a) indicated by red circles, the blue numbers refer to the submm continuum peaks as given in Table 3. The second panels display the $\mathrm{N}_{2} \mathrm{H}^{+}$column density derived from fitting the full $\mathrm{N}_{2} \mathrm{H}^{+}$hyperfine structure. The third and fourth panels show the corresponding velocity and linewidth (FWHM) of each fit. The green contours are from ATLASGAL $870 \mu \mathrm{m}$ at $0.31 \mathrm{Jy}, 0.46 \mathrm{Jy}$, and $0.61 \mathrm{Jy}$, continuing in steps of $0.3 \mathrm{Jy}$. The velocity resolution in the G15.05 map is smoothed to $0.4 \mathrm{~km} \mathrm{~s}^{-1}$ to improve the signal-to-noise ratio and increase the number of detected $\mathrm{N}_{2} \mathrm{H}^{+}$positions.

well. From the figure it can be seen that most embedded PACS sources have $\mathrm{N}_{2} \mathrm{H}^{+}$abundance ratios below $1 \times 10^{-9}$, but there seems to be no correlation between the dust temperature on scales of the beam size and the presence of embedded PACS point-sources.

\subsection{Large-scale velocity structure of clumps and filaments}

The velocity structure of the $\mathrm{N}_{2} \mathrm{H}^{+}$gas is shown in the third panel (second from the right) of Figs. 1 to 4. As explained in Sect. 2.5, we fit a single $\mathrm{N}_{2} \mathrm{H}^{+}$hyperfine structure to every pixel and display the resulting peak velocity.

The southeastern region in the map of IRDC 18182 is the IRDC in the EPoS sample. It is known that IRAS 18182-1433, originally targeted by Beuther et al. (2002a), and the IRDC have different velocities and therefore are spatially distinct. All other mapped sources show velocity variations of only a few $\mathrm{km} \mathrm{s}^{-1}$ and are therefore coherent structures.

The source with the largest spread in velocity is IRDC 18454/W43. The mapped regions in the west, beyond W43-mm1 toward W43-main (which was not mapped), have 

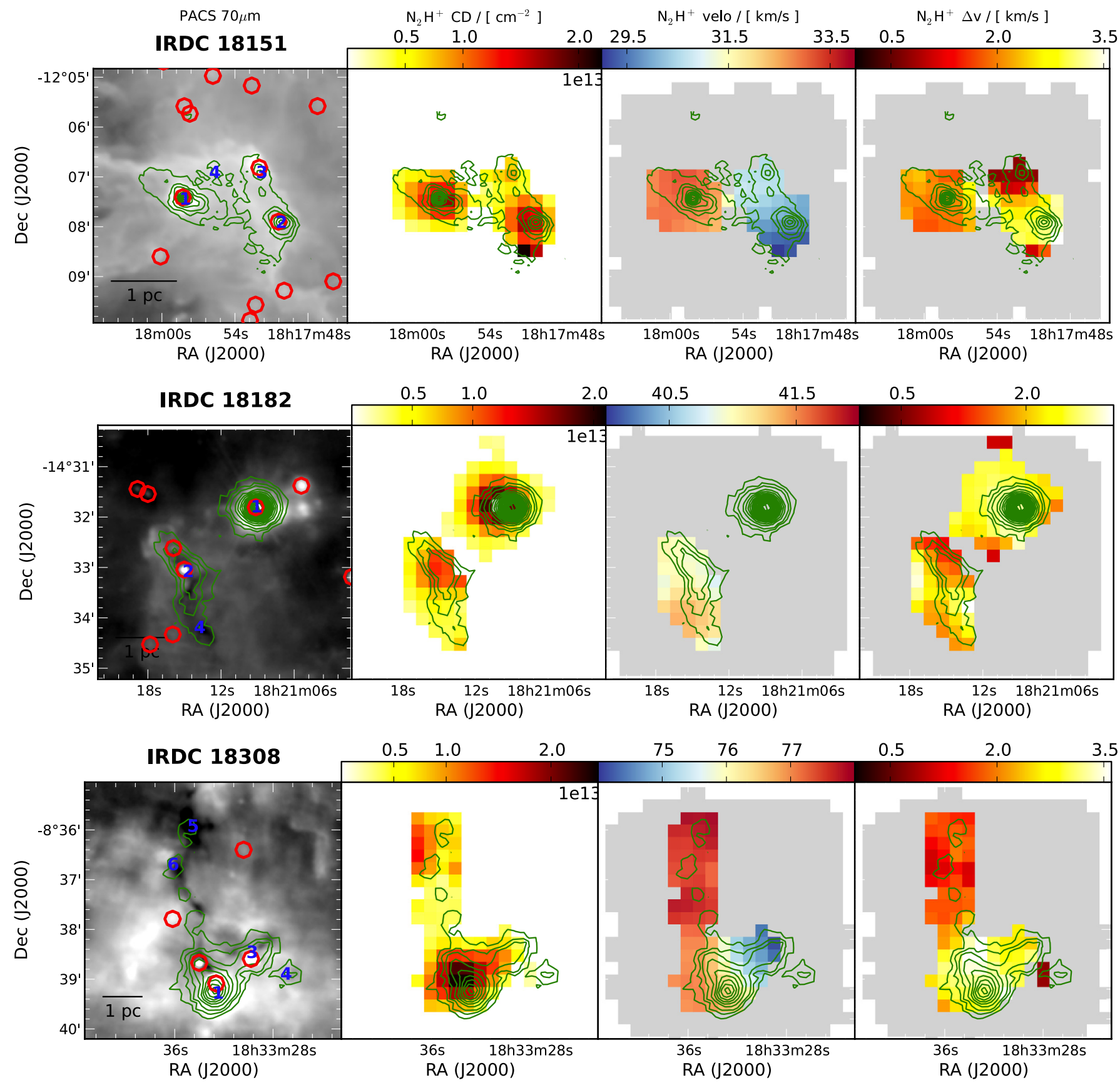

Fig. 3. Parameter maps of the regions IRDC 18151, IRDC 18182, and IRDC 18308, each mapped with the MOPRA telescope. The left panels of each row are the PACS $70 \mu \mathrm{m}$ maps with the PACS point sources detected by Ragan et al. (2012a) indicated by red circles, the blue numbers refer to the submm continuum peaks as given in Table 3. The second panels display the $\mathrm{N}_{2} \mathrm{H}^{+}$column density derived from fitting the full $\mathrm{N}_{2} \mathrm{H}^{+}$ hyperfine structure. The third and fourth panels show the corresponding velocity and linewidth (FWHM) of each fit. The green contours are from ATLASGAL $870 \mu \mathrm{m}$ at $0.31 \mathrm{Jy}, 0.46 \mathrm{Jy}$, and $0.61 \mathrm{Jy}$, continuing in steps of $0.3 \mathrm{Jy}$. For IRDC 18151 the contours are MAMBO $1.2 \mathrm{~mm}$ observations, starting at $60 \mathrm{mJy}$ in steps of $60 \mathrm{mJy}$. In all three maps the velocity resolution is smoothed to $0.4 \mathrm{~km} \mathrm{~s}^{-1}$.

the lowest velocities at below $93 \mathrm{~km} \mathrm{~s}^{-1}$, then there is a velocity gradient across W43-MM1 ending east at $97.4 \mathrm{~km} \mathrm{~s}^{-1}$, and in the far south there are two clumps at $100 \mathrm{~km} \mathrm{~s}^{-1}$. However, the velocity map was derived by fitting a single $\mathrm{N}_{2} \mathrm{H}^{+}$hyperfine structure to each spectrum. Beuther \& Sridharan (2007) and Beuther et al. (2012) have shown that at least at high spectral and spatial resolution, clump IRDC 18454-1 has two velocity components seperated by about $2 \mathrm{~km} \mathrm{~s}^{-1}$. Trying to fit each clump peak position with two $\mathrm{N}_{2} \mathrm{H}^{+}$hyperfine structures, we find that for six continuum peaks, the $\mathrm{N}_{2} \mathrm{H}^{+}$spectrum is better fitted by two independent components. For simplicity, we did not include the additional velocity component found toward IRDC 18454 at $\sim 50 \mathrm{~km} \mathrm{~s}^{-1}$ (Nguyen Luong et al. 2011).

While a more detailed description of the double-line fits are presented in Sect. 3.4, we here note that the mapped line velocity represents either a single component if one is much brighter than the other, or an average velocity of both. Therefore, the uncertainties for IRDC 18454 are significantly larger than for the other regions. Nevertheless, the large-scale velocity gradient is no artifact, but is evident in the individual spectra. 

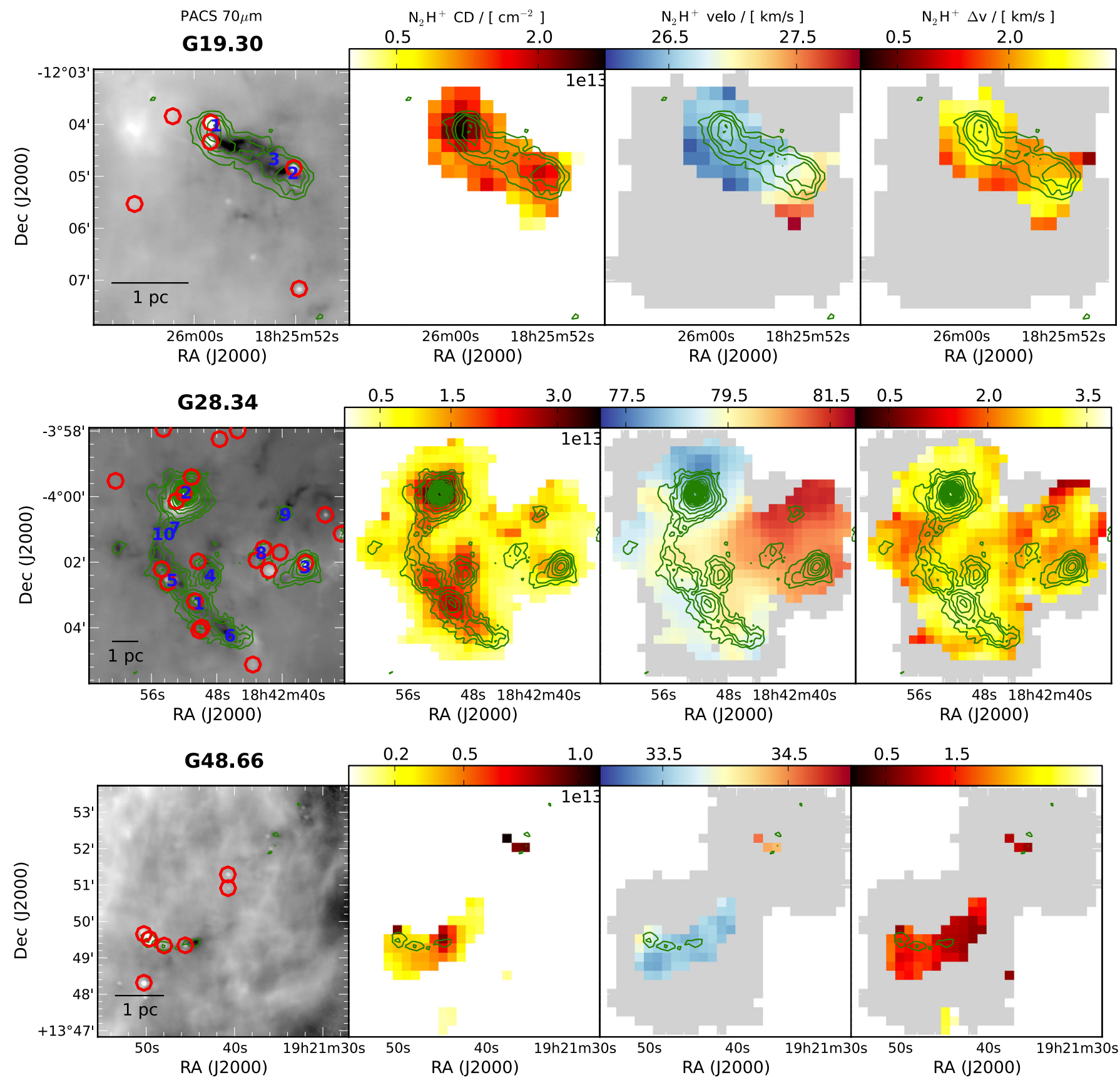

Fig. 4. Parameter maps of the regions G19.30, G28.34, and G48.66, each mapped with the MOPRA telescope. The left panels of each row are the PACS $70 \mu \mathrm{m}$ maps with the PACS point sources detected by Ragan et al. (2012a) indicated by red circles, the blue numbers refer to the submm continuum peaks as given in Table 3. The second panels display the $\mathrm{N}_{2} \mathrm{H}^{+}$column density derived from fitting the full $\mathrm{N}_{2} \mathrm{H}^{+}$hyperfine structure. The third and fourth panels show the corresponding velocity and linewidth (FWHM) of each fit. The green contours are from ATLASGAL $870 \mu \mathrm{m}$ at $0.31 \mathrm{Jy}, 0.46 \mathrm{Jy}$, and $0.61 \mathrm{Jy}$, continuing in steps of $0.3 \mathrm{Jy}$. In all three maps the velocity resolution is smoothed to $0.4 \mathrm{~km} \mathrm{~s}^{-1}$.

Studying the velocity maps in Figs. 1 through 4 in more detail, we find two different patterns of velocity structure. On the one hand, we find independent clumps that lie within the same region and may interact now or in the future, but are currently separate entities in velocity. A good example is IRDC 18151 plotted in Fig. 3. Using $870 \mu \mathrm{m}$ as dense gas tracer we resolve two clump complexes separated by $\sim 1$ pc. Across each clump there are only weak velocity variations, but the east and west complex are separated in velocity space by $3.3 \mathrm{~km} \mathrm{~s}^{-1}$. Velocity differences on the order of a few $\mathrm{km} \mathrm{s}^{-1}$ between clumps within the same structure are common and we consider such clumps as spatially connected.
On the other hand, we find smooth velocity gradients across larger structures. Clumps that may be at different velocities have connecting dense material with a continuous velocity transition in between. To exclude overlapping, but independent clumps for which either the spatial resolution or our mapping technique mimic a smooth transition we searched for double-peaked velocities and a broadening of the line width in such transition zones.

The velocity maps of G15.05, IRDC 18102, IRDC 18151 , IRDC 18182, and G48.66, Figs. 1-4 immediately reveal that these complexes have no velocity gradients in the gas above our detection limits given in Table 4 and therefore are of the first type. A summary of the clump classification is given in Table 4. 


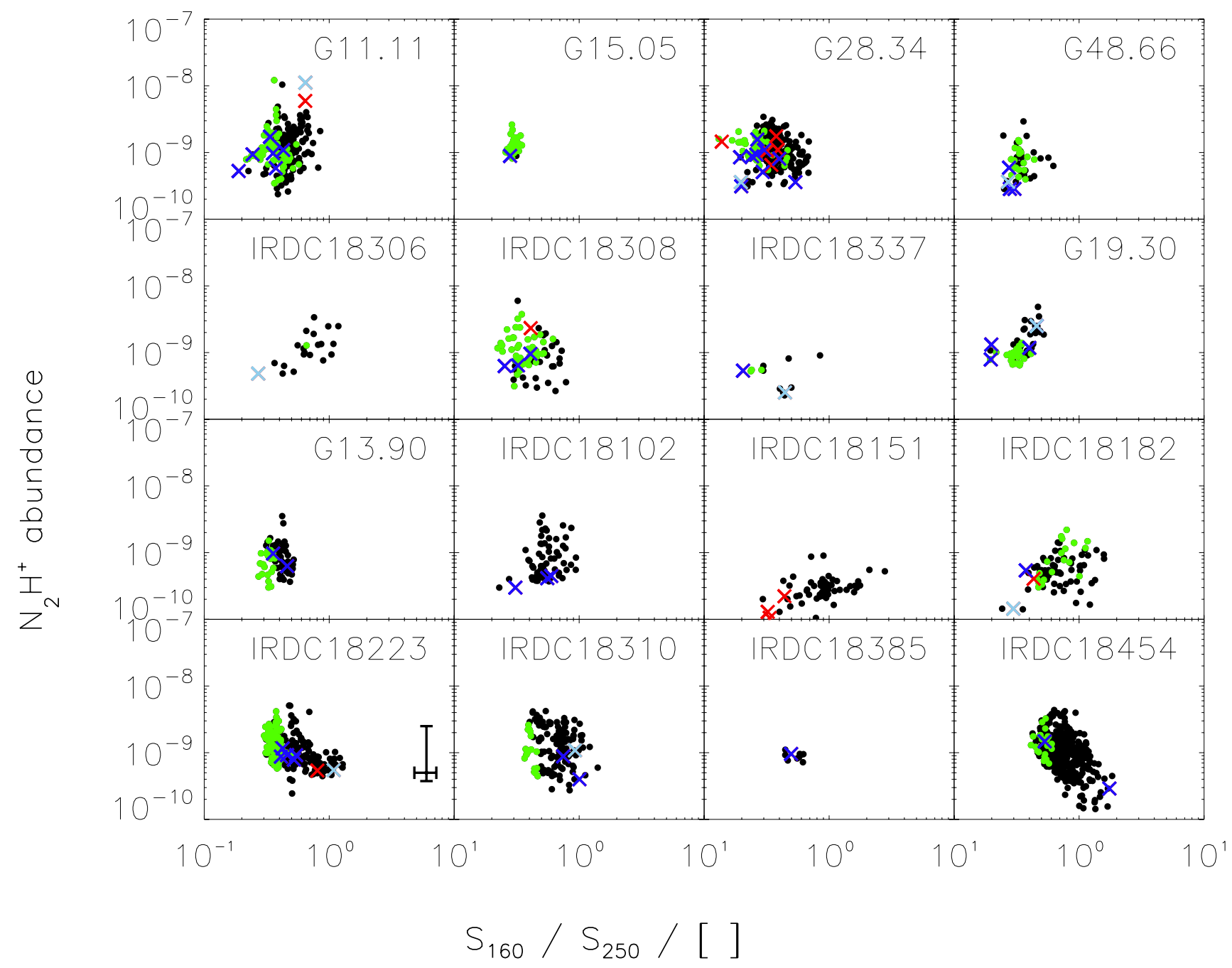

Fig. 5. $\mathrm{N}_{2} \mathrm{H}^{+}$abundance ratio over the color index between $160 \mu \mathrm{m}$ and $250 \mu \mathrm{m}$. Marked by green dots are pixels that lie within IRDCs. Overplotted with red $\times \mathrm{s}$ are all mapped PACS sources. Blue crosses also have a $24 \mu \mathrm{m}$ detection, while the light blue dots represent source that are saturated at $24 \mu \mathrm{m}$. The uncertainties given for IRDC 18223 are representative for all regions.

For IRDC 18310, shown in Fig. 1, the velocity map shows that the IRAS source in the south has a velocity of $83.2 \mathrm{~km} \mathrm{~s}^{-1}$ (see also Table 3), while the northern complex has higher velocities. Nevertheless, the velocity spread suggests an association between both clumps. In addition, the northern component itself has different velocities toward the east and south, with $86.1 \mathrm{~km} \mathrm{~s}^{-1}$ and $84.3 \mathrm{~km} \mathrm{~s}^{-1}$. In between there is a narrow transition zone with a spatially associated increase in the linewidth (see very right panel of Fig. 1). The increase in linewidth suggests that there is indeed an overlap of two independent velocity components and not a large-scale velocity transition. Using the unsmoothed Nobeyama image at a velocity resolution of $0.2 \mathrm{~km} \mathrm{~s}^{-1}$, the spectra suggest two independent components. Therefore, IRDC 18310 consists of three clumps, each showing no resolved velocity structure.

IRDC 18151, shown in Fig. 3, consists of two clumps at different velocities. While the velocities of the western clump agree within $0.5 \mathrm{~km} \mathrm{~s}^{-1}$, the eastern clump has a velocity gradient from the southeast to the northwest with a change in velocity of more than $1 \mathrm{~km} \mathrm{~s}^{-1}$. At the velocity resolution of $0.2 \mathrm{~km} \mathrm{~s}^{-1}$ we did not detect the lower column density transition region and cannot exclude a smooth transition across both clumps. To overcome the sensitivity problem we smoothed the $\mathrm{N}_{2} \mathrm{H}^{+}$data to a resolution of $0.4 \mathrm{~km} \mathrm{~s}^{-1}$. Still, only a single pixel with a good enough signal-to-noise ratio connects the two dense gas clumps. As we will discuss in Sect. 4.4, this pattern does not suggest a smooth transition.

For IRDC 18454, IRDC 18308, G11.11, G19.30, and G28.34 we found smooth velocity gradients. One of the steepest smooth velocity gradients of the sample is found toward the southern part of IRDC 18308, across the HMPO. Although there is an increase in the linewidth map, even in the unsmoothed higher resolution data we were unable to find two independent components. Across $3.2 \mathrm{pc}$ the velocity changes by $2.4 \mathrm{~km} \mathrm{~s}^{-1}$, resulting in a velocity gradient of $0.8 \mathrm{~km} \mathrm{~s}^{-1} / \mathrm{pc}$. The change in velocity occurs parallel to the elongation of the ATLASGAL $870 \mu \mathrm{m}$ emission.

The velocity gradient in the northern part of G11.11 is as clear as for IRDC 18308 and parallel to the extinction of G11.11. While the far southern tip of the northern filament has a slightly different velocity, up north it has an almost constant velocity up to the point G11.11-1 and then shows a strong but smooth gradient beyond.

As mentioned before, if considering the length and change in velocity alone, the samples steepest velocity gradient is found for IRDC 18454. Over a length of $8.4 \mathrm{pc}$ the gradient is $0.9 \mathrm{~km} \mathrm{~s}^{-1} / \mathrm{pc}$, but in between the two endpoints the velocity is not increasing monotonically. 
Table 4. Selected clump properties.

\begin{tabular}{|c|c|c|c|c|c|c|}
\hline Source & $\begin{array}{l}\text { Velocity } \\
\text { gradient }\end{array}$ & $\begin{array}{c}\text { Double } \\
\text { component }\end{array}$ & $\begin{array}{c}\text { Increase in } \Delta v \\
\text { towards peak }\end{array}$ & $\begin{array}{c}\text { Decrease in } \Delta v \\
\text { towards peak }\end{array}$ & $\begin{array}{c}\text { Outflow } \\
\text { dominated } \Delta v\end{array}$ & $\begin{array}{c}\text { Flow along } \\
\text { filament }\end{array}$ \\
\hline \multirow[t]{2}{*}{ IRDC 18223} & $\boldsymbol{S}^{1}$ & & $18223-2$ & $18223-1$ & $18223-3^{3}$ & $?$ \\
\hline & & & & 18223-1 & $18223-3$ & \\
\hline IRDC 18310 & $x$ & & & $18310-4$ & & \\
\hline IRDC 18454 & $\checkmark$ & $\checkmark$ & & & & ? \\
\hline IRDC 18102 & $x$ & & 18102 & & 18102 & \\
\hline IRDC 18151 & $x$ & & $18151-2$ & $18151-1$ & $18151-2^{3}$ & \\
\hline IRDC 18182 & $x$ & & 18182-1 & 18182-2 & & \\
\hline IRDC 18308 & $\checkmark$ & & & $18308-1$ & & $\checkmark$ \\
\hline \multirow[t]{2}{*}{ IRDC 19.30} & $\checkmark$ & & G19.30-1 & G19.30-2 & G19.30-1 $1^{3}$ & $\checkmark$ \\
\hline & & & & & G19.30- $2^{3}$ & \\
\hline \multirow[t]{2}{*}{ IRDC 11.11} & $\checkmark$ & $\checkmark$ & & G11.11-1 & G11.11-1 ${ }^{2}$ & $\checkmark$ \\
\hline & & & & G11.11-2 & & \\
\hline IRDC 15.05 & $x$ & & & & G15.05 & \\
\hline \multirow[t]{2}{*}{ IRDC 28.34} & $\checkmark$ & & G28.34-1 & G28.34-10 & $?$ & \\
\hline & & & G28.34-2 & & & \\
\hline IRDC 48.66 & $x$ & & & & G48.66 3 & \\
\hline
\end{tabular}

Notes. The columns are as follows: full region name; flag indicating whether we found a smooth velocity gradient along the region; flag indicating the presence of resolved independent velocity components along a line of sight; two columns for clumps for which we found a clear increase in linewidth toward the center and toward the edge clumps, respectively; flag indicating that the velocity profile is consistent with flows along the filament. ${ }^{(1)}$ For 18223 we found several velocity gradients, both along and perpendicular to the filament. ${ }^{(2)}$ The angle between the outflow and the linewidth broadening does not match exactly. ${ }^{(3)}$ Indirect evidence for outflow (mainly from SiO), but the direction of the outflow is unknown.

IRDC 18223 shows significant changes in the velocity field, but is not listed among the clumps with smooth velocity gradients. The changes of the velocity are on $0.5 \mathrm{pc}-1 \mathrm{pc}$ scales and show no clear pattern. Nevertheless, at the given velocity resolution of $0.5 \mathrm{~km} \mathrm{~s}^{-1}$ we found no overlapping independent $\mathrm{N}_{2} \mathrm{H}^{+}$components. Therefore, all the gas on the scales we trace seems to be connected. It is worth noting that the two southern clumps, IRDC 18223-2 and IRDC 18223-3, have a gradient along the short axis of the filament, which might be interpreted as evidence of rotation. Velocities in the east are higher than in the west. In contrast, although less well mapped, the IRAS source in the north, IRDC 18223-1, has a velocity gradient along the short filament axis as well, but in opposite direction; velocities in the west are higher than in east.

For G13.90, IRDC 18385, IRDC 18306, and IRDC 18337 we lack the sensitivity to draw a conclusion. While IRDC 18385 , and IRDC 18306 are very poorly mapped, for G13.90 and IRDC 18337 we mapped the main emission structures, but with the available sensitivity we did not trace the gas in between the dense clumps. In both sources, the detected clumps have different velocities, with a gradient across the clumps in IRDC 18337. Since we did not trace the gas in between the dense clumps, we are unable to assess whether the velocity transitions are smooth, or if the clumps have no connection in velocity space.

\section{4. $\mathrm{N}_{2} \mathrm{H}^{+}$linewidth in the context of young PACS sources and column density peaks}

The right panels of Figs. 1 to 4 show the fitted linewidth (FWHM) for the mapped regions. The distribution of the linewidth is very different for each region and density peak. While it increases toward some of the submm peaks (e.g. IRDC 18102, IRDC 18182-1), for others the peak of the linewidth is on the edge of the submm clumps (e.g. IRDC 18223-1, IRDC 18223-3, G19.30). The IRDCs for which we detect $\mathrm{N}_{2} \mathrm{H}^{+}$and that have no embedded/detected PACS source often have a narrower linewidth than other clumps of the same region with embedded protostars.

A brief description of the linewidth distribution of each region is given in Appendix A. In the following we discuss a few interesting or notable examples.

While the linewidth in IRDC 18223 significantly increases toward IRDC 18223-2, the linewidth toward IRDC 18223-1, a well-studied HMPO (Sakai et al. 2010), and IRDC 18223-3, an object known to drive a powerful outflow (Beuther \& Sridharan 2007; Fallscheer et al. 2009), increases toward the edges of the dust continuum. Compared with other regions of IRDC 18223, the linewidth at IRDC 18223-3 is broader, but it becomes even broader in the northwest. This aligns very well with the outflow found by Fallscheer et al. (2009) and can be explained by it (for the outflow direction see the first row of Fig. 1, right panel). IRDC 18223-1 was originally identified as IRAS 18223-1243 and is bright at IR wavelengths (down to $K$ band). However, typical tracers of ongoing high-mass star formation such as $\mathrm{cm}$ emission, water and methanol masers, or SiO-tracing shocks are not detected (Sridharan et al. 2002; Sakai et al. 2010). Only the CO line wings found by Sridharan et al. (2002) are indicative of outflows, which might explain the bipolar broadening of the $\mathrm{N}_{2} \mathrm{H}^{+}$linewidth. Nevertheless, despite its prominence at IR wavelengths and with the luminosity of the PACS point source at its peak of $2000 L_{\odot}$ (point source 8 in Ragan et al. 2012a), the linewidths at the continuum peak are not exceptional within this region. In contrast, although IRDC 18223-2 is detected at near IR wavelengths as well and the PACS point source at its center has a luminosity of only $200 L_{\odot}$, the linewidth is $2.5 \mathrm{~km} \mathrm{~s}^{-1} \mathrm{com}$ pared with $1.9 \mathrm{~km} \mathrm{~s}^{-1}$ for IRDC 18223-1. Because Beuther \& Sridharan (2007) found no SiO toward IRDC 18223-1/2 we exclude a strong outflow, and the reason for the line broadening is not clear at all. Thus one should keep in mind that IRDC 18223-2 has not been investigated in such great detail and we cannot entirely exclude an outflow.

We excluded IRDC 18454 from the analysis of the linewidth because, as mentioned in Sect. 3.3, we found multiple velocity 


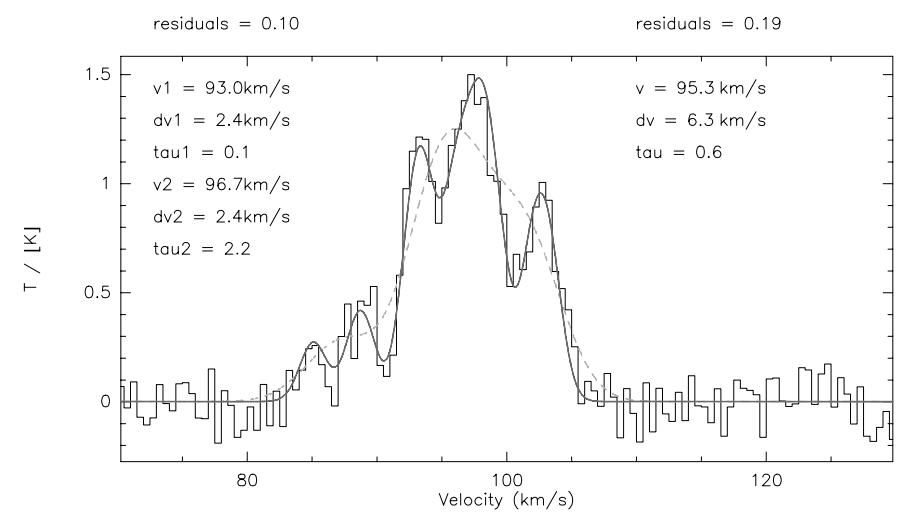

Fig. 6. Spectra of IRDC 18454-4 Beuther \& Sridharan (2007); Beuther et al. (2012). While the dashed line shows the single-component fit with the fitting parameters to the right, the solid line is the two-component fit with its fitting parameters to the left. The residuals are the results of the minimize task in CLASS.

components toward several positions. Figure 6 displays an example of an $\mathrm{N}_{2} \mathrm{H}^{+}$spectrum that compares a single-component fit with a double-component fit. Comparing the residuals of the two different fits as calculated by CLASS, for the six clumps in which we found two independent components the residuals are on average reduced by $30 \%$. For all two-component fits, the linewidth decreases compared with a single-component fit. Still, the linewidths are on average broader than for the other clumps listed in Table 3.

Similar double velocity component fits toward the peaks are otherwise only possible in G11.11. Here, eight of the clumps are fit better by two independent $\mathrm{N}_{2} \mathrm{H}^{+}$components. Different from IRDC 18454, the linewidth of the two components becomes on average narrower than the linewidth of other clumps in the sample. In addition, the improvement of the residuals is only $20 \%$. Therefore it is unclear whether two independent components are present or if the fit is simply improved because of the larger number of free parameters. A systematic study of the multiple components is beyond the scope of this paper.

For G13.90, IRDC 18385, IRDC 18306, and IRDC 18337 the mapped areas are not sufficient to draw conclusions.

Similar to Fig. 5, Fig. 7 shows the relation between the linewidth (FWHM) of $\mathrm{N}_{2} \mathrm{H}^{+}$and the color index. Because the color index is a proxy of the temperature, one might expect a correlation between these two quantities, but there is no correlation at all. Figure 8 plots the $\mathrm{N}_{2} \mathrm{H}^{+}$linewidth versus the $\mathrm{H}_{2}$ column density, but again, we find no correlation.

In the context of the linewidth and dust mass, the virial analysis can be used to understand whether structures are gravitationally bound or are transient structures. Following MacLaren et al. (1988), we calculated the virial mass of our clumps via $M_{\text {vir }}=k R \Delta v^{2}$. For the clump radius $R$ we used the effective radius calculated by CLUMPFIND. The geometrical parameter $k$ depends on the density distribution, with $k=190$ for $\rho \sim r^{-1}$, and $k=126$ for $\rho \sim r^{-2}$. Beuther et al. (2002a), Hatchell \& van der Tak (2003), and Peretto et al. (2006) found typical density distributions in sites of massive star formation of $\rho \propto r^{\alpha}$ with $\alpha \sim-1.6$, in between both parameters. While we list the virial mass for both parameters in Table 3, we used the intermediate value of $k=158$ in Fig. 9.

The $\alpha$ parameter as defined in Bertoldi \& McKee (1992) is the ratio of the internal kinetic energy and the gravitational energy. Their virial parameter as defined in Eq. (2.8a) of Bertoldi $\&$ McKee (1992) $\left(\alpha=\frac{5 \sigma^{2} R}{G M}\right)$ without another geometrical parameter resembles a spherical distribution of constant density. Because of the geometrical correction factor we applied to the mass calculations, the presented virial parameters are smaller by a factor of 1.32. A histogram of the virial parameter is plotted in Fig. 9.

If we assume the error on our linewidth to be lower than $15 \%$, the uncertainties of the calculated virial mass are mainly determined by the geometrical parameter $k$. The actual error on the given virial masses is significantly larger since the calculation neglects all physical effects but gravity and thermal motions (kinetic energy). For the conceptual quantity we can neglect these effects and estimate the error to be $\sim 50 \%$.

\section{Discussion of $\mathrm{N}_{2} \mathrm{H}^{+}$dense gas properties}

In the following, we discuss the kinematic properties of the sources we mapped in $\mathrm{N}_{2} \mathrm{H}^{+}$, as described above.

\subsection{Dense clumps and cores}

The clump masses in the range of several tens of $M_{\odot}$ to a few thousands of $M_{\odot}$ show that most regions have the potential to form massive stars in the future, or show signs of ongoing highmass star formation. One should keep in mind that the listed peak column densities are averaged over the beam. As has been shown by Vasyunina et al. (2009) assuming an artifical $r^{-1}$ density profile, true peak column densities are higher by a factor of 20 to 40. This agrees with interferometric observations of clumps within our sample (Beuther et al. 2005, 2006; Fallscheer et al. 2011). Therefore, all peak column densities become higher than $3 \times 10^{23} \mathrm{~cm}^{-2}$, or $1 \mathrm{~g} / \mathrm{cm}^{2}$. This reinforces the view that the mapped clumps are capable of forming massive stars. The high column densities also agree with the detection of $\mathrm{N}_{2} \mathrm{H}^{+}$as highdensity gas tracer.

\subsection{Abundance ratios}

To understand why the abundance of $\mathrm{N}_{2} \mathrm{H}^{+}$is expected to vary with embedded sources or temperature, one needs to understand the formation mechanism. The formation of $\mathrm{N}_{2} \mathrm{H}^{+}$works via $\mathrm{H}_{3}^{+}$which also builds the basis for the formation of $\mathrm{HCO}^{+}$from $\mathrm{CO}$. Because of the high abundance of $\mathrm{CO}$ in cold dense clouds, the production of $\mathrm{HCO}^{+}$is initially dominant and consumes all $\mathrm{H}_{3}^{+}$. If during cloud contraction the temperatures become cold enough for $\mathrm{CO}$ to freeze out, $\mathrm{N}_{2} \mathrm{H}^{+}$can be produced more efficiently and eventually becomes more abundant than $\mathrm{HCO}^{+}$. The situation changes again when $\mathrm{CO}$ is released from the grains either due to heating or due to shocks. The CO destroys the $\mathrm{N}_{2} \mathrm{H}^{+}$ and forms $\mathrm{HCO}^{+}$instead, making $\mathrm{HCO}^{+}$more abundant again. (For a more detailed discussion see Jørgensen et al. 2004.)

In summary, the early (more diffuse) cloud phase is dominated by $\mathrm{HCO}^{+}$, while the quiet dense clumps should be dominated by $\mathrm{N}_{2} \mathrm{H}^{+}$. With the onset of star formation, $\mathrm{HCO}^{+}$is becoming dominant again.

The EPoS sample mainly has been selected to cover regions of ongoing, but early star formation. For this $\mathrm{N}_{2} \mathrm{H}^{+}$line survey, we selected regions covering all evolutionary stages. Many of them have both infrared-quiet regions at the wavelengths range covered previous to Herschel and well-known and luminous IRAS sources. Together with the Herschel data, hardly any region of high column density is genuinely infrared-dark.

As a result of the $\mathrm{N}_{2} \mathrm{H}^{+}$evolution and the broad range of evolutionary stages covered, we expect a wide range of $\mathrm{N}_{2} \mathrm{H}^{+}$ 
J. Tackenberg et al.: Flows along massive star-forming regions

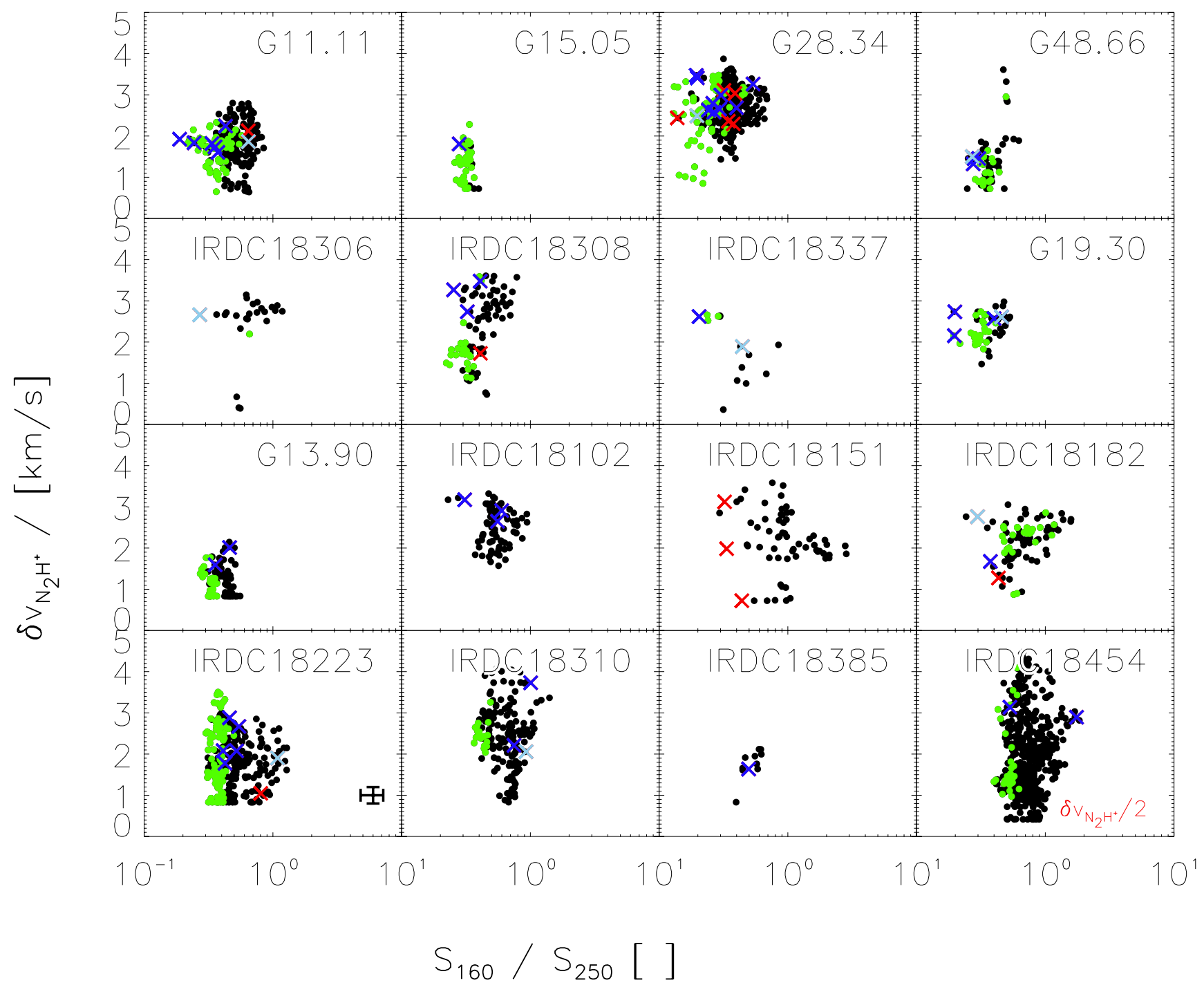

Fig. 7. $\mathrm{N}_{2} \mathrm{H}^{+}$linewidth versus the color index for the $160 \mu \mathrm{m}$ over the $250 \mu \mathrm{m}$ band. Marked by green dots are pixels that lie within IRDCs. Overplotted with red Xs are all PACS sources that were mapped. Blue dots also have a $24 \mu \mathrm{m}$ detection, while the pale blue dots represent sources that are saturated at $24 \mu \mathrm{m}$. The uncertainties given for IRDC 18223 are representative for all regions. For IRDC 18454 the linewidths were multiplied by a factor of 0.5 to fit the data points into the plotting range.

abundance ratios. As has been discussed in Sect. 3.2, Fig. 5 shows the correlation between the $\mathrm{N}_{2} \mathrm{H}^{+}$abundance and the $160 \mu \mathrm{m}$ to $250 \mu \mathrm{m}$ flux ratio as a proxy of the temperature. For all regions, the bulk of all pixels has $\mathrm{N}_{2} \mathrm{H}^{+}$abundances ratios of $1 \times 10^{-9}$. This agrees well with earlier studies of high-mass star-forming regions (Vasyunina et al. 2011, and references therein). At the same time, several regions (e.g. G11.11, G28.34, IRDC 18454) show abundance variations of two orders of magnitude. While this is a result of the various evolutionary stages within each region, it is worth noting that it seems to be uncorrelated to the flux ratio of $160 \mu \mathrm{m}$ over $250 \mu \mathrm{m}$.

To correlate some areas with an evolutionary stage, in Fig. 5 we mark regions that show up in extinction at $70 \mu \mathrm{m}$ by green dots. As Fig. 5 shows, these regions are among the coldest within each region. Nevertheless, high $\mathrm{N}_{2} \mathrm{H}^{+}$abundances are found not only in IRDCs or cold regions. In contrast to IRDC pixels which mark the earliest and coldest evolutionary stages, the PACS-only sources mark regions in which star formation is about to start (red), and the MIPS bright PACS sources indicate ongoing star formation (blue). All pixels connected to a PACS source have low $\mathrm{N}_{2} \mathrm{H}^{+}$abundances. Whether this is due to an increase in temperature or probably to shocks is unclear.

It has been shown in Ragan et al. (2012a) that sources with a detected $24 \mu \mathrm{m}$ counterpart are on average warmer, more luminous, and more massive and that therefore a $24 \mu \mathrm{m}$ counterpart is indicative of a more evolved source. Nevertheless, the PACS core properties in Ragan et al. (2012a) show a large overlap between MIPS bright and dark sources. Therefore, one cannot draw a clear conclusion on the evolutionary stage (temperature, luminosity, or mass) based on a $24 \mu \mathrm{m}$ detection alone. This easily explains the exceptions, for instance, in G11.11.

\subsection{Signatures of overlapping dense cores within clumps}

We have described in Sect. 3.3 two independent velocity components toward six of the IRDC 18454 continuum peaks, as well as seven clumps in G11.11 with double-peaked $\mathrm{N}_{2} \mathrm{H}^{+}$lines. The two components have velocity offsets of only a few $\mathrm{km} \mathrm{s}^{-1}$. Since the hyperfine structure of $\mathrm{N}_{2} \mathrm{H}^{+}$includes an optically thin component, we can exclude opacity and self-absorption effects, a common feature in dense star-forming regions. The two 


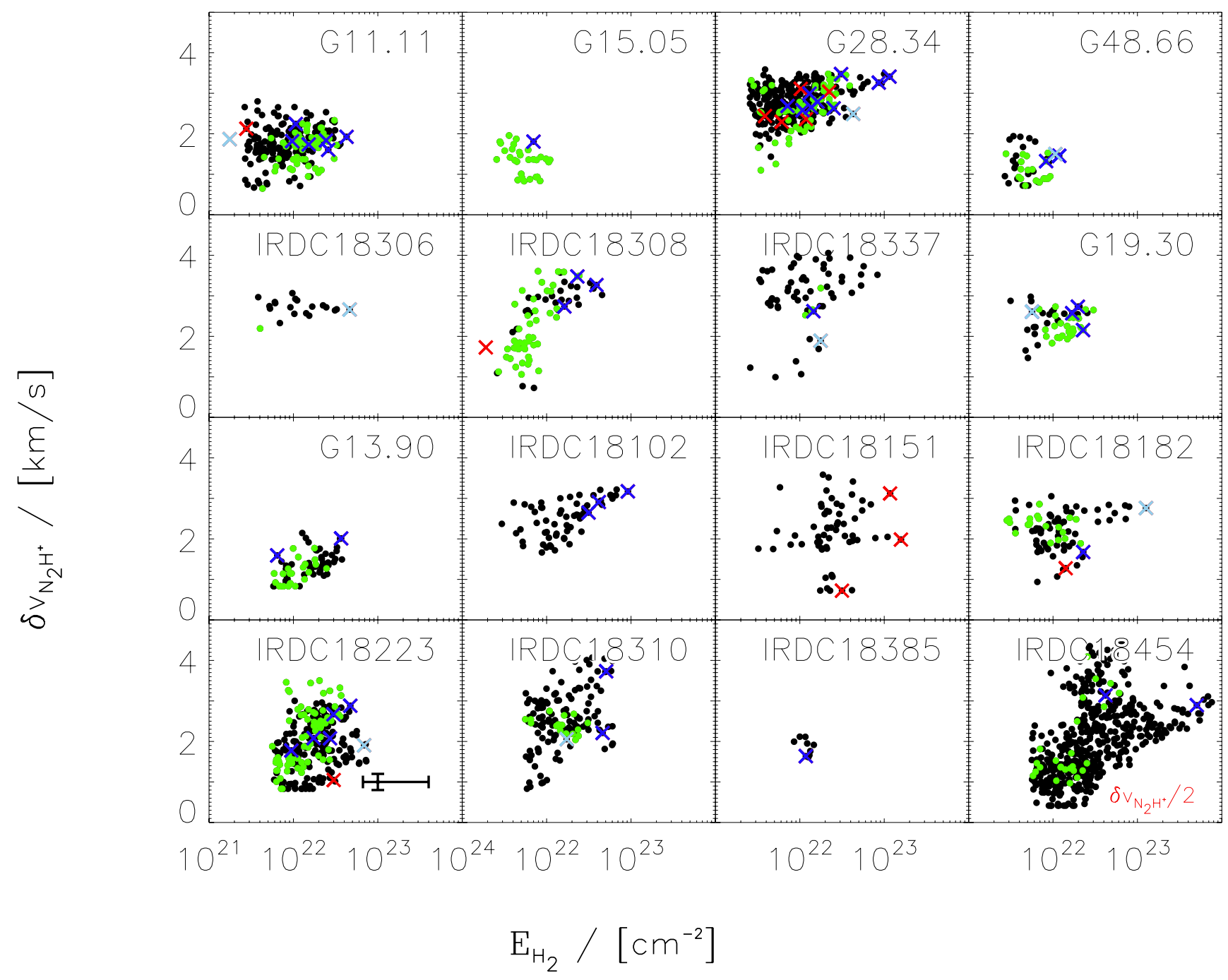

Fig. 8. Plot of the $\mathrm{N}_{2} \mathrm{H}^{+}$linewidth versus the dust column density. Marked by green dots are pixels that lie within IRDCs. Overplotted with red Xs are all PACS sources that were mapped. Blue dots also have a $24 \mu \mathrm{m}$ detection, while the pale blue dots represent sources that are saturated at $24 \mu \mathrm{m}$. The uncertainties given for IRDC 18223 are representative for all regions. For IRDC 18454 the linewidths were multiplied by a factor of 0.5 to fit the data points into the plotting range.

independent velocity components within IRDC 18454 have previously been reported by Beuther \& Sridharan (2007); Beuther et al. (2012) and Ragan et al. (in prep.) found multiple velocity components toward G11.11. Combining our $\mathrm{N}_{2} \mathrm{H}^{+}$Nobeyama data with PdBI observations at $\sim 4^{\prime \prime}$, Beuther et al. (2013) revealed multiple independent velocity components toward IRDC 18310-4. These are not resolved within the Nobeyama data alone at the spatial resolution of $18^{\prime \prime}$. Similar multicomponent velocity signatures have been found in high spatial resolution images of dense cores in Cygnus-X (Csengeri et al. 2011a,b) and toward IRDCs by Bihr et al. (2014). Therefore, it seems to be a common feature in high-mass star-forming regions.

Using radiative transfer calculations of collapsing high-mass star-forming regions, Smith et al. (2013) showed that such double-peaked line profiles may be produced by the superposition of infalling dense cores. Therefore, in high-resolution studies, which filter out the large-scale emission, multiple cores along the line of sight can be detected. However, comparing our beam sizes of $\sim 0.5 \mathrm{pc}$ for IRDC 18454 and $\sim 0.8 \mathrm{pc}$ for G11.11 to typical sizes of cores below $0.1 \mathrm{pc}$, the larger-scale clump gas is expected to probably dominate our signal. Therefore it is clear that multiple velocity components due to cores are more likely to be identified in high spatial resolution imaging. While IRDC 18454 is at the intersection of the spiral arm and the Galactic bar, and therefore exceptional in many aspects, G11.11 is most likely a more typical high-mass star-forming region, similar to what has been simulated by Smith et al. (2009) and Smith et al. (2013). If the double-peaked line profiles originate from two dense cores within our beam, as suggested by Smith et al. (2013), the cores within G11.11 would need to be extremely dense or large. Instead, it seems more realistic that we detect the gas of the clump as one velocity component, and the second component is produced by an embedded single core of high-density contrast moving relative to its parent clump. For IRDC 18454 we found double velocity spectra even inbetween the peak positions. This suggests that the components are coming from two overlapping sheets that are close in velocity. It is unclear whether these sheets are interacting or not.

\subsection{Accretion flows along filaments?}

In Sect. 3.3 we presented the velocity structure of the $16 \mathrm{ob}$ served high-mass star-forming regions. As we described, five complexes have no velocity structure, while six regions have 
J. Tackenberg et al.: Flows along massive star-forming regions
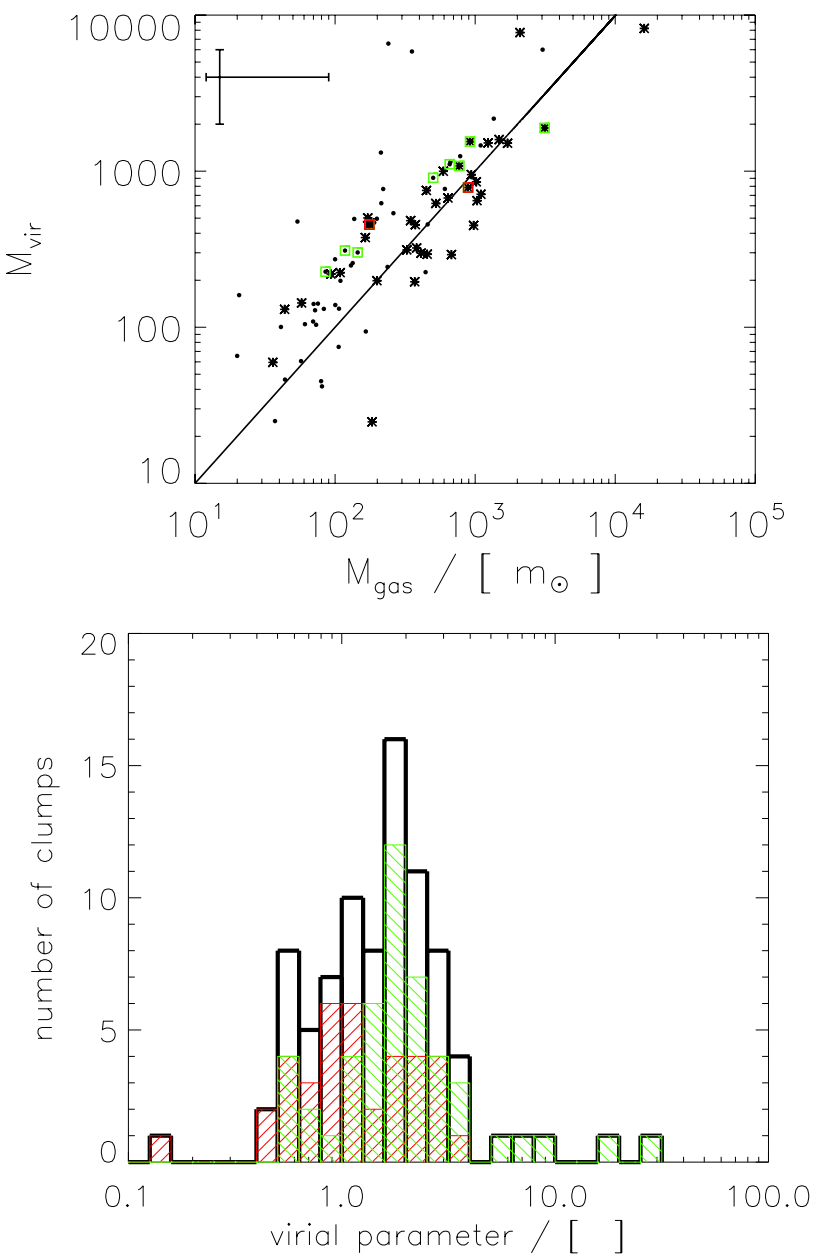

Fig. 9. Top panel: virial mass derived from the $\mathrm{N}_{2} \mathrm{H}^{+}$linewidth over the gas mass. The virial mass assumes a geometrical parameter of $k=158$, which is intermediate between $k=126$ for $1 / \rho^{2}$ and $k=190$ for $1 / \rho$. While the black dots indicate clumps without a PACS point source inside, the asterisks represent clumps with a PACS point source. Marked by green and red squares are the clumps of G28.34, with green boxes representing clumps in global infall, and red boxes representing clumps with signatures of outward moving gas (for details see Tackenberg et al. 2014). The solid line indicates unity. Lower panel: histogram of the virial parameter $\alpha$. While the black histogram represents the full sample, the red and green histogram is the subset of clumps with and without a PACS point source, respectively.

smooth large-scale velocity gradients. The velocity structure of IRDC 18223 is more complex and does not fit into either of these categories. For four regions we lack the sensitivity to draw a conclusion.

Despite the two general appearances, the large-scale velocity structure of the clumps is very diverse. In general, structures larger than $1 \mathrm{pc}$ usually show some velocity fluctuations. These can be either steady and smooth, or pointing to separate entities. It is worth noting that the physical resolution of the $\mathrm{N}_{2} \mathrm{H}^{+}$observations ranges from $0.1 \mathrm{pc}$ to $1.0 \mathrm{pc}$, with an average of $0.3 \mathrm{pc}$ for the $18^{\prime \prime}$ Nobeyama beam, and 0.7 pc for the $46^{\prime \prime}$ with MOPRA. Therefore, we are unable to resolve smaller structures, and the $1 \mathrm{pc}$ limit is observationally set. In fact, velocity fluctuations on smaller scales are still likely. However, the observations show that on the clump scale, some clumps do show gas motions, while others are kinematically more quiescent. High-resolution studies, for example that of Ragan et al. (2012b), have proven for some regions that gas motions continue on smaller scales.

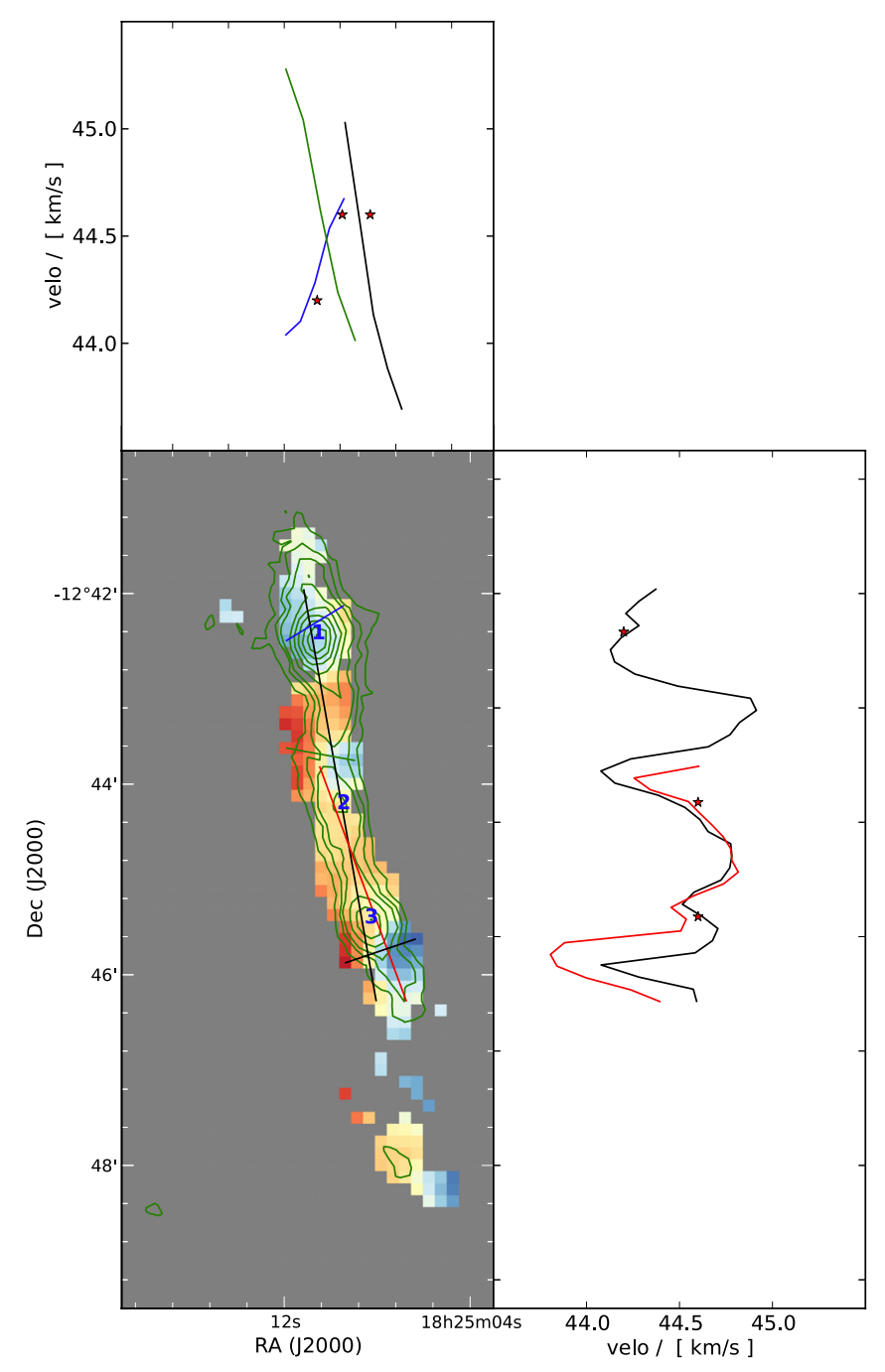

Fig. 10. Profile of the $\mathrm{N}_{2} \mathrm{H}^{+}$velocity of IRDC 18223. The left panel shows the velocity map with contours from ATLASGAL superimposed (see also Fig. 1). The right and top panels show the velocity cuts along the lines marked on the velocity map. The stars mark the velocities of the clump peaks.

To understand the velocity structure of complexes with smooth velocity transitions, Figs. 10 through 16 visualize the velocity gradients along given lines. As discussed in Sect. 3.3, our velocity map of IRDC 18151 consists of two larger structures, IRDC 18151-1 in the east, and IRDC 18151-2 and IRDC 181513 in the west. The overall changes within the eastern and western clump are $\sim 0.5 \mathrm{~km} \mathrm{~s}^{-1}$ and $\sim 1 \mathrm{~km} \mathrm{~s}^{-1}$, respectively. While the velocity cut through the eastern clump shows hardly any variation, the western clump has a noticeable velocity gradient. To detect at least part of the gas at intermediate velocities, we smoothed the $\mathrm{N}_{2} \mathrm{H}^{+}$to a velocity resolution of $0.4 \mathrm{~km} \mathrm{~s}^{-1}$. Figure 12 shows the velocity profile across both clumps. While the western clump shows a slight velocity increase toward the east, the eastern clump shows no velocity gradient. In particular, the two gradients seem not to match, and if they interact dynamically, the transition zone would need to be short. Therefore we conclude that the two structures are individual components, but in the context of other dense gas tracers they seem to be embedded within the same cloud. If seen from a slightly different angle, the double velocity components discussed in Sect. 4.3 could well originate from such a structure. 


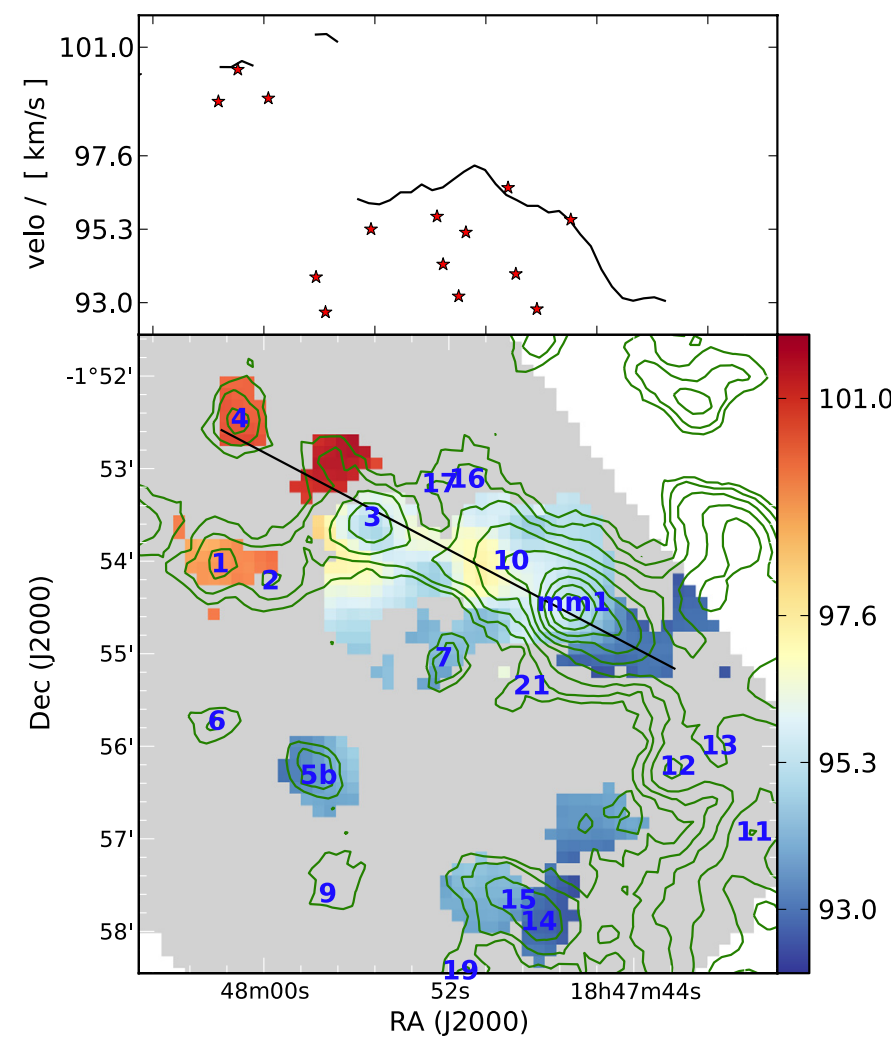

Fig. 11. Profile of the $\mathrm{N}_{2} \mathrm{H}^{+}$velocity of IRDC 18454. The left panel shows the velocity map with contours from ATLASGAL superimposed (see also Fig. 1). The top panel shows the velocity cut along the line marked on the velocity map. The stars mark the velocities of the clump peaks.

\subsubsection{Flows along G11.11}

We found a clear smooth transition of the velocity toward the northern part of G11.11. Shown by the top profile in Fig. 14, between G11.11-1 and G11.11-12, the differences in velocity are below $0.5 \mathrm{~km} \mathrm{~s}^{-1}$. Along the profile just south of G11.11-1, the velocity starts to increase, with higher velocities toward G11.112 and beyond. On the other hand, the profiles perpendicular to the filament (right panel of Fig. 14) have almost constant velocities. Only the profile closest to G11.11-1 has a velocity gradient, but the filament has a bend exactly at the position of the profile. A profile perpendicular to the actual shape of the IRDC would have no velocity gradient. Therefore, we conclude that the velocity gradient occurs solely along the filament.

Both Tobin et al. (2012, observationally), and Smith et al. (2013, numerically) suggested large-scale accretion flows along filaments on, and probably producing, central cores. They described the expected observational signatures for filaments that are inclined from the plane of the sky. Imagine a cylinder with a central core and material flowing onto the core from both sides at a constant velocity. For simplicity and without loss of generality, we set the central core at rest. Then the gas has a constant velocity along the filament on each side of the central object. Because the gas is flowing in from both sides onto the core, a constant velocity is observed for both directions. The angle of the filament to the line of sight determines the observed velocity component. While Tobin et al. (2012) accounted for a gravitationally accelerated gas flow that is expected to have a velocity jump at its center, the synthetical observations of highmass star-forming regions performed by Smith et al. (2013) have

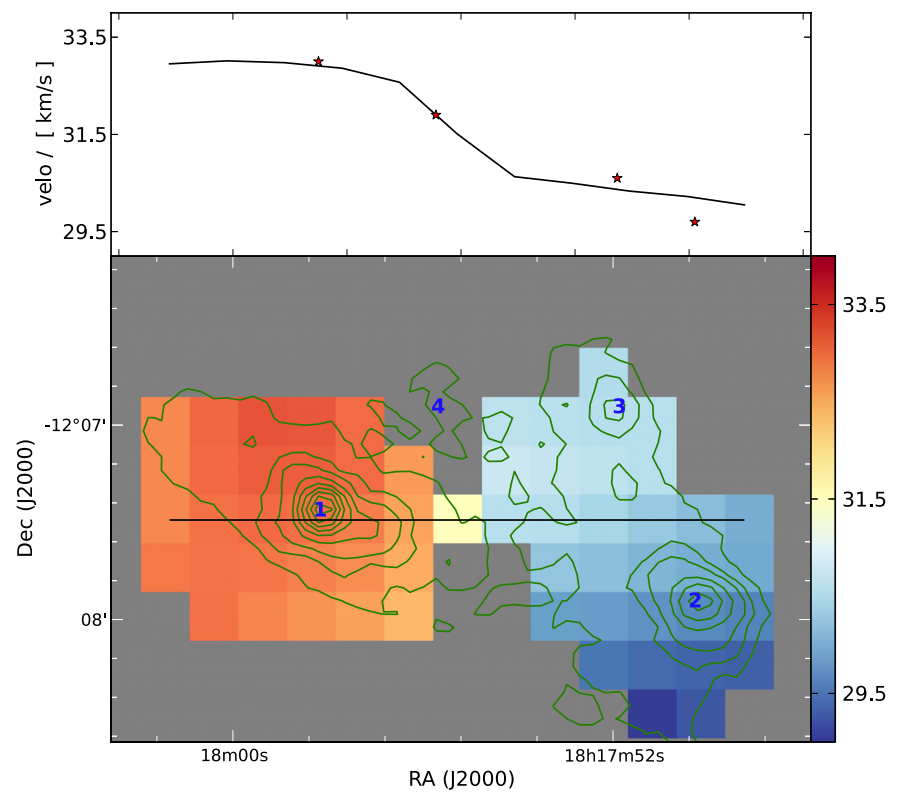

Fig. 12. Profile of the $\mathrm{N}_{2} \mathrm{H}^{+}$velocity of IRDC 18151. The left panel shows the velocity map with contours from ATLASGAL superimposed (see also Fig. 3). The top panel shows the velocity cut along the line marked on the velocity map. The stars mark the velocities of the clump peaks.

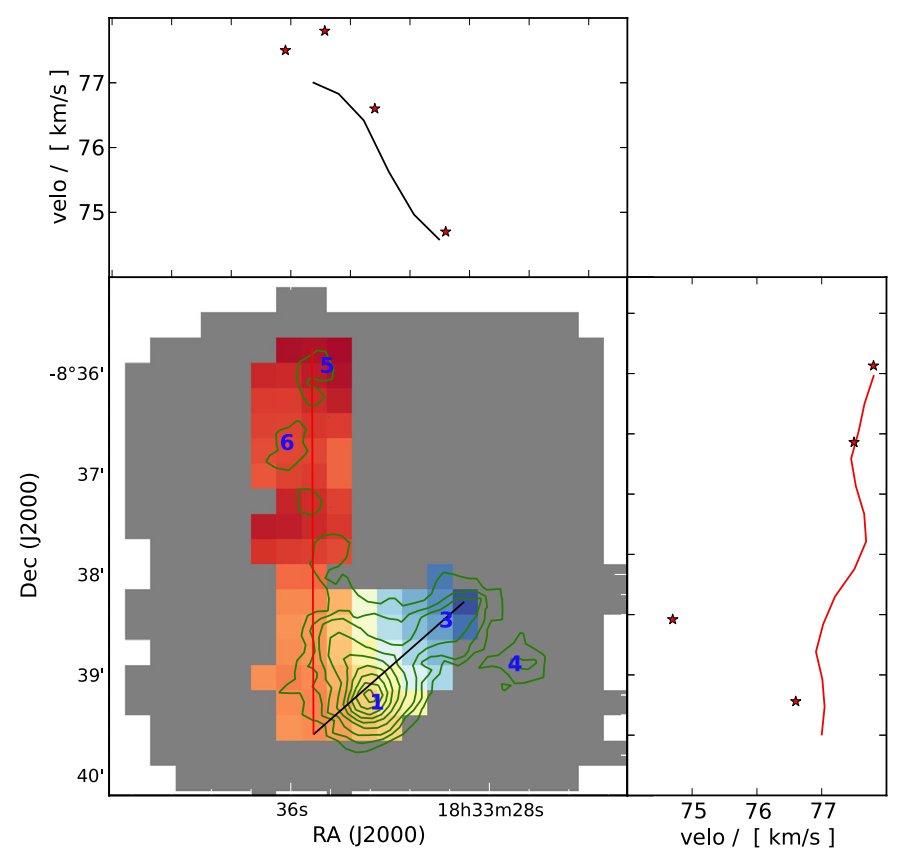

Fig. 13. Profile of the $\mathrm{N}_{2} \mathrm{H}^{+}$velocity of IRDC 18308. The left panel shows the velocity map with contours from ATLASGAL superimposed (see also Fig. 3). The top and right panels show the velocity cuts along the lines marked on the velocity map. The stars mark the velocities of the clump peak.

a smooth transition. These authorts also found local velocity variations connected to smaller substructures.

Recalling the velocity structure we found for G11.11, our observations may be explained by such accretion flows along the filament. The filament would need to have an angle such that the southeast is farther away from us than the northwest. The almost constant velocity over $\sim 3$ pc would be material moving toward G11.11-1, the most massive clump in the region. Just 
J. Tackenberg et al.: Flows along massive star-forming regions

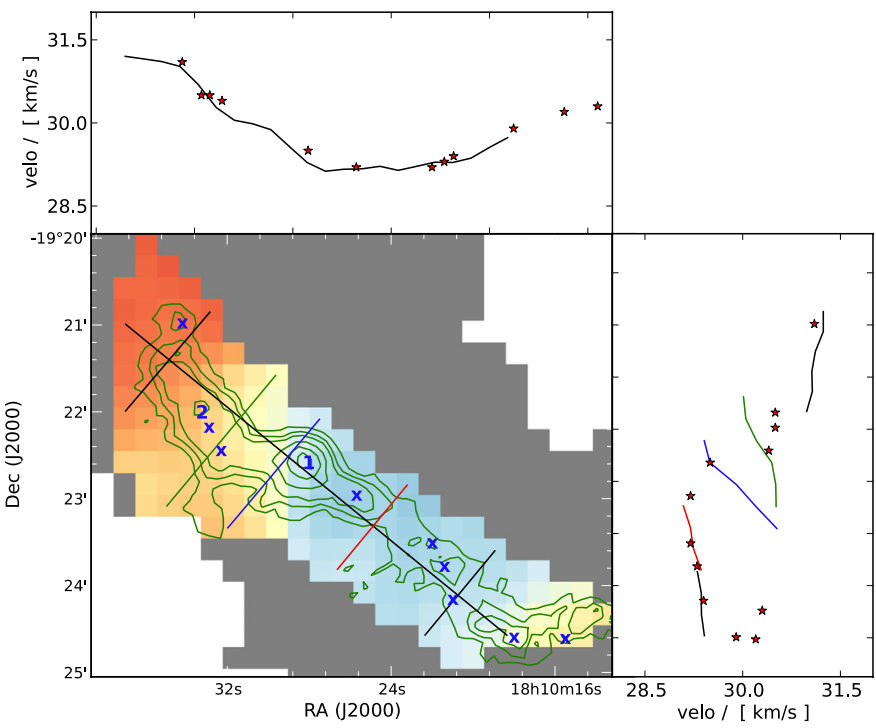

Fig. 14. Profile of the $\mathrm{N}_{2} \mathrm{H}^{+}$velocity of the northern part of G11.11. The left panel shows the velocity map with contours from ATLASGAL superimposed (see also Fig. 2). The right and top panels show the velocity cuts along the lines marked on the velocity map. The stars mark the velocities of the clump peak.

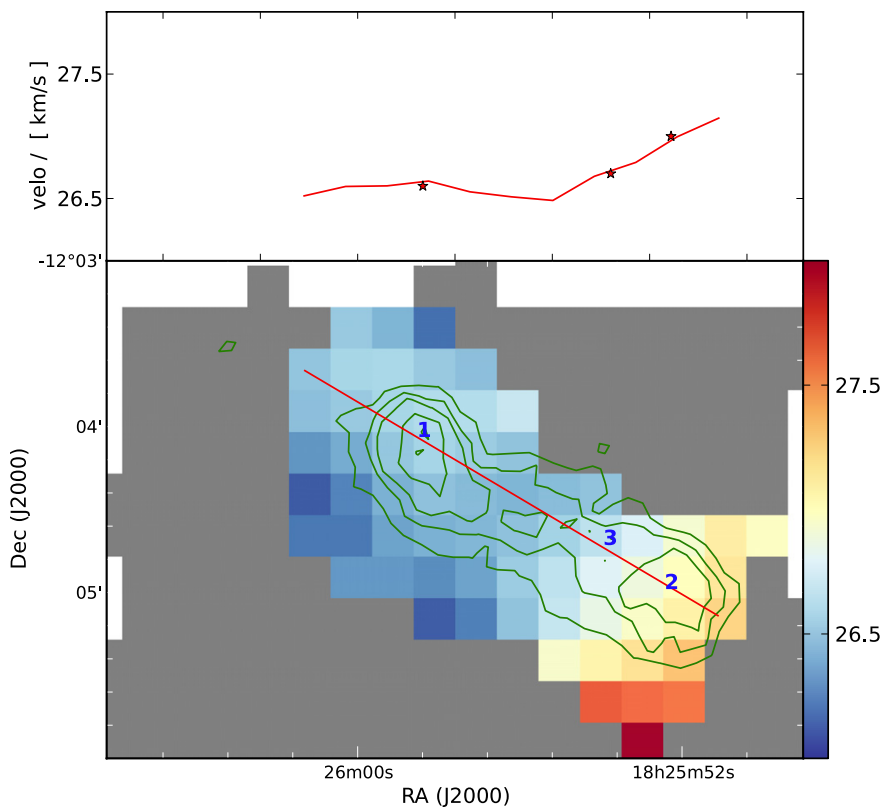

Fig. 15. Profile of the $\mathrm{N}_{2} \mathrm{H}^{+}$velocity of G19.30. The left panel shows the velocity map with contours from ATLASGAL superimposed (see also Fig. 4). The top panel shows the velocity cut along the line marked on the velocity map. The stars mark the velocities of the clump peak.

before G11.11-1, the velocity starts to increase and we observe the transition across the clump. Beyond G11.11-1, the gravitational potential of G11.11-2, the second-most massive clump in this region, accretes material on its own, and accelerates the gas even farther beyond the position of G11.11-1.

The scales we trace are an order of magnitude larger than what has been discussed by Smith et al. (2013) and our resolution is an order of magnitude lower. Because of the second dense clump, we do not observe the theoretically predicted pattern. The increase in velocity may also be explained by solid-body rotation of part of the filament. Nevertheless, we propose an accretion flow along the filament as a possible explanation for the

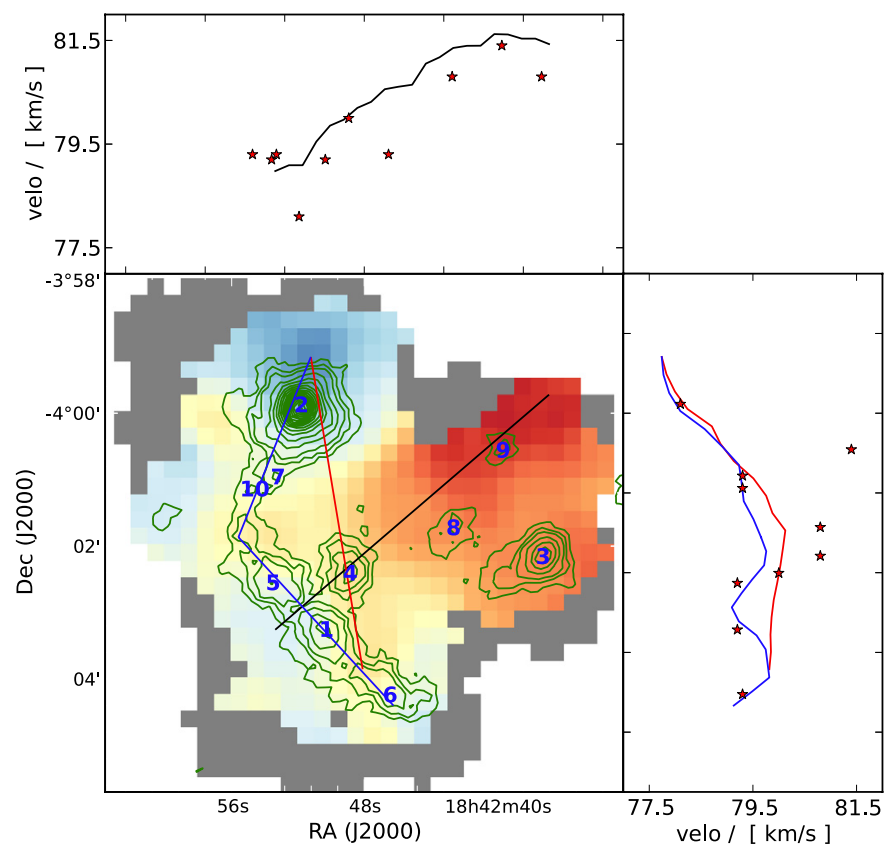

Fig. 16. Profile of the $\mathrm{N}_{2} \mathrm{H}^{+}$velocity of G28.34. The left panel shows the velocity map with contours from ATLASGAL superimposed (see also Fig. 4). The top and right panels show the velocity cuts along the lines marked on the velocity map. The stars mark the velocities of the clump peaks.

observed velocity pattern in G11.11. This view is supported by the fact that star-formation is most active at the center of the potential infall.

Consequently, if high-mass star formation is actively ongoing within G11.11-1, the material flow along the filament suggests continuous feeding of the mass reservoir from which forming stars can accrete.

\subsubsection{Flows along IRDC 18308}

A similar scenario might explain the velocity pattern along the IR-dark part of IRDC 18308. The cut along the IRDC ending at the HMPO (Fig. 13) shows only minor changes in velocity across the IRDC of $\sim 2.5 \mathrm{pc}$ length. In the vicinity of IRDC $18308-1$, the velocity changes by almost $1 \mathrm{~km} \mathrm{~s}^{-1}$ on a short physical scale of only $\sim 0.6 \mathrm{pc}$. The cut does leave a gap to the HMPO and does not fully close up in velocity. As described in Sect. 3.3, across the HMPO we found one of the steepest velocity gradients in our sample, but the origin is unclear. One possible explanation that would produce a similar velocity profile is solid-body rotation. In this picture, the knees at both ends of the profile would be caused by a transition from solid-body rotation to viscous rotation because of the lower densities in the outer regions. A full explanation would require a combination of hydrodynamic simulations with radiative transfer calculations. This is beyond the scope of this paper.

\subsubsection{Flows along $\mathrm{G} 19.30$ ?}

In G19.30, along the northeastern part of the IRDC, the velocity is constant over $\sim 1 \mathrm{pc}$ and then rises toward its other end. This suggests that the gas is flowing across G19.30-1, at the northeastern end, through G19.30-3 toward G19.30-2. Interestingly, G19.30-1 is the most massive clump and therefore is potentially building the center of gravity. Therefore, the 
flow is opposite to the gravitational potential. The dust temperatures derived for the cores within G19.30 by Ragan et al. (2012a) are higher for the more massive clump G19.30-1, which increases our uncertainties on the mass. Nevertheless, even if we assume the higher dust temperature of $25 \mathrm{~K}$ for the whole G19.30-1 clump, and a temperature of $17 \mathrm{~K}$ for G19.30-2, the two masses become of the same order. The additional clump G19.30-3 close to G19.30-2, is of similar mass as G19.30-2 and therefore increases the gravitational potential of the southwestern end. But even if our interpretation of a gas flow along the filament were correct, we found no evidence that is it driven by gravity. Instead, this example might be interpreted as an indication for a primordial origin of the flows, which leads to the formation of the clumps. Similar as for G11.11, the flows along the filaments within G19.30 and IRDC 18308 support the idea that the mass reservoir in high-mass star formation is continuously replenished.

\subsubsection{The peculiar case of IRDC 18223}

IRDC 18223 is filamentary, but with a more complex velocity structure than the previously discussed regions. The velocity seems to be oscillating along the filament. The emission peaks of the southern clumps, IRDC 18223-2 and IRDC 18223-3, are at the same velocity of $44.6 \mathrm{~km} \mathrm{~s}^{-1}$ and in between the variations are minor. The peak of the clump IRDC 18223-1, harboring the HMPO IRAS 18223-1243, has a velocity of $44.2 \mathrm{~km} \mathrm{~s}^{-1}$.

Perpendicular to the filament, we found three extreme velocity gradients, shown in Fig. 10. Although they do not pass exactly across the dust continuum emission peaks, each seems to be associated with a clump. A straightforward interpretation would be solid-body rotation along the filament axis. But as mentioned in Sect. 3.3, the two lower profiles indicate rotation in the direction opposite to the uppermost profile. In Sect. 3.4 we discussed the possible influence of the powerful outflow within IRDC $18223-3$ on the linewidth distribution. The same outflow could also alter the velocity distribution in its direct vicinity. Nevertheless, a change in rotation orientation along a single filament seems counterintuitive. For the massive filament DR21 within Cygnus X, Schneider et al. (2010) found three velocity gradients perpendicular to the filament axis, with alternating directions. Suggesting turbulent colliding flows as origin of the filament, these authors interpreted the velocity pattern as a remnant of the external flow motions.

Within IRDC 18223-3, on scales of 7", Fallscheer et al. (2009) found a velocity gradient in $\mathrm{N}_{2} \mathrm{H}^{+}$and $\mathrm{CH}_{3} \mathrm{OH}$, a highdensity and shocked-gas tracer. They successfully modeled the $\mathrm{CH}_{3} \mathrm{OH}$ velocity gradient with a rotating and infalling toroid that was significantly larger than the massive-disk candidates (e.g. Cesaroni et al. 2005; for an example of the opposite see Boley et al. 2012). However, the-small scale velocity gradient presented in Fallscheer et al. (2009) not only has a slightly different orientation (by $\sim 45^{\circ}$ ), but is also rotating in the oppsite direction to the gradient presented in Fig. 10. This implies that the two gradients are independent and have different origins; the small-scale infalling toroid does not connect the large-scale envelope to a possibly even smaller-scaled disk. To first order this contradicts our previous statement of continuously fed clumps because in this particular case, the large-scale mass reservoir, or envelope, seems to be disconnected from the embedded HMPO. On the one hand, the velocity gradient seen in the large-scale gas might be confused with the outflow. As we discussed in Sect. 4.5.1, there is a clear correlation between the outflow and the linewidth distribution. Although $\mathrm{N}_{2} \mathrm{H}^{+}$is not known to be commonly entrained in outflows, a similar correlation between the outflow and the velocity gradient seems probable and might explain the change in velocity. Here we need to emphasize that the outflow direction and velocity gradient have an angle of almost $90^{\circ}$. On the other hand, it may well be that the accretion phase of IRDC 18223-3 has already stopped, and the largescale envelope and the inner toroidal structure are decoupled. But apart from its youth, it is counterintuitive to assume that the core first accreted from a rotating structure and then developed an internal source rotating in the opposite direction. Therefore, we cannot offer a good explanation.

\subsubsection{G28.34}

A quite large and complex region is G28.34. Most prominent and clear, it has a strong but smooth east-to-west velocity gradient. Along the velocity cut given in the top panel of Fig. 16, the velocity changes by $2.6 \mathrm{~km} \mathrm{~s}^{-1}$ over $7.1 \mathrm{pc}$. This results in a velocity gradient of $0.4 \mathrm{~km} \mathrm{~s}^{-1} / \mathrm{pc}$. The clump emission peaks within the southeastern component of G28.34, namely G28.346 , G28.34-1, and G28.34-5, all have the same velocity. As the velocity cut along them shows, the velocity of the dense gas between them seems to be oscillating, similar as found for IRDC 18223. Even farther north, the emission peaks of G28.347, and G28.34-10 are still at the same velocity as the more southern clumps. Only north of them the velocity changes monotonically to lower velocities on the order of a parsec.

From the smooth velocity transition from east to west and from the absence of double-peaked $\mathrm{N}_{2} \mathrm{H}^{+}$profiles, we conclude that all dense gas within the region is physically connected. To fully explain the observed velocity signatures of the complex dense gas morphology in G28.34, numerical simulations are most likely required as well.

\subsubsection{IRDC 18454}

The velocity analysis of IRDC 18454 is hampered because, as discussed in Sect. 4.3, several clumps show two independent velocity components at their peak position. Therefore, the mapped single velocities can either be the stronger of the two components, or an average of both. However, the large-scale velocity gradient in east-to-west direction is clear and no artifact.

\subsection{Discussion of the linewidth distribution}

\subsubsection{Outflow-induced turbulence}

As we have described in Sect. 3.4, we found no correlation between the linewidth and the temperature or $\mathrm{H}_{2}$ column density.

The lack of a correlation between the temperature and the linewidth can be explained by the narrow thermal linewidth. At $20 \mathrm{~K}$, the thermal line-broadening of $\mathrm{N}_{2} \mathrm{H}^{+}$is $\sim 0.18 \mathrm{~km} \mathrm{~s}^{-1}$ (in FWHM), and $\sim 0.16 \mathrm{~km} \mathrm{~s}^{-1}$ at $15 \mathrm{~K}$. Compared with the observed linewidths of a few $\mathrm{km} \mathrm{s}^{-1}$, the nonthermal contribution dominates by far and temperature variations of a few $\mathrm{K}$ are weaker than our uncertainties of the measured FWHM.

Another possible contribution to the linewidth stems from turbulence. Because the clouds are close to virial equilibrium (see Fig. 9), the linewidth is expected to scale with the column density. As mentioned in Sect. 3.4, we found no correlation between these two quantities either.

Instead, the spatial resolution of the data prefers molecular outflows as the dominating contribution to the measured linewidths. Examining the $\mathrm{N}_{2} \mathrm{H}^{+}$linewidth maps of each region, 
we found clear imprints of known outflows onto the linewidth distribution. A good example in this context is IRDC 182233, shown in Fig. 1. As we described in Sect. 3.4, Fallscheer et al. (2009) reported the outflow direction in IRDC 18223-3 to be $\sim 135^{\circ}$ east of north. As Fig. 1 shows, we observe a line broadening along the same axis, with the highest velocities toward the edge. Due to the complex overall velocity structure it is unclear whether the aligned velocity gradient is connected to the outflow as well. This would suggest the origin of the increased linewidth to be an unresolved velocity gradient. In contrast, shocks connected to bipolar outflows could enhance the turbulence, leading to broader lines. Another possible origin of a linewidth-broadening toward the edge of clumps is provided by gravo-turbulent fragmentation, as described in Klessen et al. (2005). Therefore, we cannot assess the physical origin of the linewidth distribution. In this particular case the alignment of outflow and linewidth broadening suggests a direct connection.

A similarly clear correlation between the linewidth and outflow we found for IRDC 18102, for which the outflow direction has been observed by Beuther (priv. comm.). For G11.11-1, Gómez et al. (2011) observed an outflow in east-west direction, or $90^{\circ}$ east of north, while we found a significant line-broadening similar to a bipolar outflow with an angle of $\sim 150^{\circ}$ east of north. This means that the angle between outflow and increase in linewidth is on the order of $60^{\circ}$. Therefore, a direct correlation between the two observations is not mandatory, but possible in the context of the other regions.

Although the outflow has not been spatially resolved, the presence of $\mathrm{SiO}$ suggests the existence of an outflow toward the northern part of G15.05, while the southern part is SiO-quiet (Linz et al., in prep.). Even though both the northern and southern part are IR-dark even at $70 \mu \mathrm{m}$ and the mass distribution peaks in between, the linewidth distribution in the north is significantly larger than in the south. A possible outflow might explain this linewidth distribution. Using $\mathrm{SiO}$ as a general tracer of outflows, we found a similar correspondence between increased linewidth and the presence of outflows for IRDC 181512, G19.30-1, and G19.30-2, and the eastern tip of G48.66.

No $\mathrm{SiO}$ was found toward the IR dark part of IRDC 18182, IRDC 18182-2, and -4, for which the linewidths are among narrowest within the region. In between the two clumps the linewidth broadens without the presence of known outflows. Therefore, additional factors have to be dominating here, for example, superposition of the two independent velocity components.

The four pointings toward $\mathrm{G} 28.34$ that cover $\mathrm{SiO}$ emission (Linz et al., in prep.) agree with the picture of an outflowdominated linewidth distribution. Nevertheless, due to the generally broad lines in that region, the variations are less pronounced than in all other regions.

A particular case seems to be IRDC 18151-1. Fallscheer et al. (2011) reported an outflow connected to it with an angle of $315^{\circ}$ east of north, but the linewidth is narrower than in IRDC $18151-2,1.9 \mathrm{~km} \mathrm{~s}^{-1}$ compared with $3.1 \mathrm{~km} \mathrm{~s}^{-1}$, respectively. In addition, it shows hardly any linewidth structure, and therefore differs from the other clumps discussed so far.

Sridharan et al. (2002) and Sakai et al. (2010) found no SiO toward the two northern clumps of IRDC 18223, IRDC 182231, and IRDC 18223-2, but Sridharan et al. (2002) interpreted the detection of $\mathrm{CO}$ line wings as an indication for an outflow. Therefore, the bipolar line-broadening of the edges of IRDC 18223-1 (see Sect. 3.4) might be caused by an outflow. In contrast, the linewidth broadens toward the center of IRDC 18223-2. Because the large-scale velocity gradient across
IRDC 18223-2 is twice as steep as for IRDC 18223-1, the linebroadening toward the center could be explained by unresolved small-scale velocity gradients. An alternative explanation for the broadening of the linewidth could be colliding flows, as discussed in Sect. 4.4.4. However, the velocity gradients are not perfectly aligned with the linewidth broadening, which would be expected for colliding flows. We have no information on the $\mathrm{SiO}$ or other outflow tracers for IRDC 18310, and IRDC 18308.

\subsubsection{The particular case IRDC 18454}

The line-width map of IRDC 18454 for a single-component fit (Fig. 1) provides a combination of the two detected velocity components (see Sec. 4.3). Therefore, it quantifies the internal motions within each beam. If the two velocity components originate from two sheets that are interacting, a combined linewidth is appropriate for the virial analysis. However, resolving the individual velocity components at the peak positions significantly reduces the line width of the clumps. They become more similar to the other regions within this survey, but still, the resulting line widths are broader than the average line width that we found for all other clumps.

Combining our Nobeyama $45 \mathrm{~m} \mathrm{~N}_{2} \mathrm{H}^{+}$single-dish data with observations from the Plateau de Bure interferometer, Beuther et al. (2012) found two independent velocity components toward IRDC 18454-1 as well. While one component is consistent in both width and velocity with one of our velocity components, the other component is offset by $\sim 1 \mathrm{~km} \mathrm{~s}^{-1}$ and twice as wide in the single-dish data. This difference can be explained by the better spatial resolution of $\sim 3.5^{\prime \prime}$ of the PdBI observations. Although the two fragments, which Beuther et al. (2012) resolved within IRDC 18454-1, have very similar velocities, Fig. 7 in their paper shows a velocity spread of a few $\mathrm{km} \mathrm{s}^{-1}$ over the Nobeyama beam size of $18^{\prime \prime}$. As already apparent from Fig. 1, the linewidth toward IRDC 18454-1 is particularly narrow compared with almost all other clumps within IRDC 18454 . This can partly be explained by the absence of any $70 \mu \mathrm{m}$ PACS source and its physical proximity to $\mathrm{W} 43-\mathrm{mm} 1$.

\subsection{Discussion of the virial analysis}

In the context of the virial analysis, bound clumps are expected because most clumps show signs of ongoing early star formation or already have formed dense cores. Nevertheless, in globally unbound regions collapsing fragments might exist as well and form stars and clusters. As shown in Fig. 9, most clumps have larger virial masses than gas masses, or a slightly positive $\alpha$ index. Still, within the errors all clumps might be gravitationally bound. Marked in the upper panel of Fig. 9 are all clumps associated with G28.34. As we will discuss in a second paper (Tackenberg et al. 2013), G28.34 is in a state of global infall. Nevertheless, despite their global contraction, their virial parameters are not different from the bulk of the distribution. This again emphasizes that a virial mass larger than the gas mass does not necessarily lead to dissolving clumps.

From Fig. 9 it appears that clumps without a PACS point source have a higher $\alpha$ parameter than clumps with a PACS point source. Furthermore, more massive clumps tend to have lower $\alpha$ parameters. Here it is worth noting that clumps with PACS point sources are on average more massive than clumps without embedded sources. In the context of both, flows along the filaments as discussed here and global infall as discussed in Tackenberg et al. (2013), the mass of clumps increases over 
time. The difference between the populations with and without a PACS point source can accordingly be explained to first order by two simple scenarios. (1) The dense material that we observe today accumulated and fragmented on a very short time-scale. Suggested by the competitive-accretion scenario, some clump fragments accrete mass faster than others. Therefore, they became dense enough to start forming embedded sources earlier. (2) In an alternative scenario, not all clumps formed at the same time. Assuming a similar mass-accretion rate for all clumps, the oldest clumps are the densest ones today, and have already started to form stars.

\section{Conclusions}

Complementary to the existing Herschel/EPoS data, we mapped 17 massive star-forming regions in $\mathrm{N}_{2} \mathrm{H}^{+}$. Using CLUMPFIND on submm data, mainly from the ATLASGAL survey at $870 \mu \mathrm{m}$, we extracted the clump population that our observations cover. Assuming a constant dust temperature of $20 \mathrm{~K}$ and the distances given in Ragan et al. (2012a), we calculated both peak column densities and clump masses. With only a few exceptions, the $\mathrm{N}_{2} \mathrm{H}^{+}$and total gas column density distribution agrees very well spatially, but the $\mathrm{N}_{2} \mathrm{H}^{+}$abundance varies in IRDCs by two orders of magnitude.

While five complexes show no velocity structure, six regions have smooth velocity gradients. For three regions the velocity structure is consistent with accretion flows along a filament. For DR 21 Schneider et al. (2010) found a velocity pattern very similar to what we found for IRDC 18223 and suggested that the velocity gradients perpendicular to the filament are remnants of colliding flow motions from which the filaments formed. For G28.34 and IRDC 18454, the velocity structure is very complex and a detailed modeling is beyond the scope of this work.

The linewidth distribution among the sources is very diverse; in particular, IRDC 18454 stands out. Even after resolving double-peaked line profiles and treating them carefully, its average linewidth is twice the value we found for all other regions. This might be explained by the location of IRDC 18454, which is associated with W43, a mini-starburst at the junction of a spiral arm and the Galactic bar. Sources for which the outflow direction is known show a line-broadening along the outflow. In addition, all clumps that have measured $\mathrm{SiO}$ show an increased linewidth, while the other clumps show hardly any variations. Therefore, the linewidth on the scale of clumps probably is dominated by outflows and unresolved velocity gradients.

In conclusion, this study supports a very dynamic starformation scenario. Clumps and cores accrete mass from the dense filaments. It is unclear whether the cores and filaments form from primordial flows or if the clump gravitational potentials are the origin of the flows.

Acknowledgements. We are grateful to the referee for her/his constructive comments. S. E. R. is supported by grant RA 2158/1-1, which is part of the Deutsche Forschungsgemeinschaft priority program 1573 ("Physics of the Interstellar Medium"). PACS has been developed by a consortium of institutes led by MPE (Germany) and including UVIE (Austria); KU Leuven, CSL, IMEC (Belgium); CEA, LAM (France); MPIA (Germany); INAF-IFSI/OAA/OAP/OAT, LENS, SISSA (Italy); IAC (Spain). This development has been supported by the funding agencies BMVIT (Austria), ESA-PRODEX (Belgium), CEA/CNES (France), DLR (Germany), ASI/INAF (Italy), and CICYT/MCYT (Spain). SPIRE has been developed by a consortium of institutes led by Cardiff University (UK) and including Univ. Lethbridge (Canada); NAOC (China); CEA, LAM (France); IFSI, Univ. Padua (Italy); IAC (Spain); Stockholm Observatory (Sweden); Imperial College London, RAL, UCL-MSSL, UKATC, Univ. Sussex (UK); and Caltech, JPL, NHSC, Univ. Colorado (USA). This development has been supported by national funding agencies: CSA (Canada); NAOC (China); CEA,
CNES, CNRS (France); ASI (Italy); MCINN (Spain); SNSB (Sweden); STFC (UK); and NASA (USA).

\section{References}

André, P., Men'shchikov, A., Bontemps, S., et al. 2010, A\&A, 518, L102 Barnard, E. E. 1919, ApJ, 49, 1

Battersby, C., Bally, J., Ginsburg, A., et al. 2011, A\&A, 535, A128

Benjamin, R. A., Churchwell, E., Babler, B. L., et al. 2003, PASP, 115, 953

Bertoldi, F., \& McKee, C. F. 1992, ApJ, 395, 140

Beuther, H., \& Sridharan, T. K. 2007, ApJ, 668, 348

Beuther, H., Schilke, P., Menten, K. M., et al. 2002a, ApJ, 566, 945

Beuther, H., Schilke, P., Menten, K. M., et al. 2002b, in Hot Star Workshop III: The Earliest Phases of Massive Star Birth, ed. P. Crowther, ASP Conf. Ser., 267, 341

Beuther, H., Sridharan, T. K., \& Saito, M. 2005, ApJ, 634, L185

Beuther, H., Zhang, Q., Sridharan, T. K., Lee, C.-F., \& Zapata, L. A. 2006, A\&A, 454,221

Beuther, H., Churchwell, E. B., McKee, C. F., \& Tan, J. C. 2007, in Protostars and Planets V (Tucson: University of Arizona Press), 165

Beuther, H., Tackenberg, J., Linz, H., et al. 2012, A\&A, 538, A11

Beuther, H., Linz, H., Tackenberg, J., et al. 2013, A\&A, 553, A115

Bihr, S., Beuther, H., Linz, H., et al. 2014, A\&A, submitted

Boley, P. A., Linz, H., van Boekel, R., et al. 2012, A\&A, 547, A88

Bonnell, I. A., Vine, S. G., \& Bate, M. R. 2004, MNRAS, 349, 735

Bronfman, L., Nyman, L.-A., \& May, J. 1996, A\&AS, 115, 81

Carey, S. J., Clark, F. O., Egan, M. P., et al. 1998, ApJ, 508, 721

Carey, S. J., Noriega-Crespo, A., Mizuno, D. R., et al. 2009, PASP, 121, 76

Cesaroni, R., Neri, R., Olmi, L., et al. 2005, A\&A, 434, 1039

Clark, P. C., Glover, S. C. O., Klessen, R. S., \& Bonnell, I. A. 2012, MNRAS, 424, 2599

Contreras, Y., Schuller, F., Urquhart, J. S., et al. 2013, A\&A, 549, A45

Csengeri, T., Bontemps, S., Schneider, N., Motte, F., \& Dib, S. 2011a, A\&A, 527, A 135

Csengeri, T., Bontemps, S., Schneider, N., et al. 2011b, ApJ, 740, L5

Egan, M. P., Shipman, R. F., Price, S. D., et al. 1998, ApJ, 494, L199

Egan, M. P., Price, S. D., Kraemer, K. E., et al. 2003, VizieR Online Data Catalog: V/114

Fallscheer, C., Beuther, H., Zhang, Q., Keto, E., \& Sridharan, T. K. 2009, A\&A, 504, 127

Fallscheer, C., Beuther, H., Sauter, J., Wolf, S., \& Zhang, Q. 2011, ApJ, 729, 66

Faúndez, S., Bronfman, L., Garay, G., et al. 2004, A\&A, 426, 97

Goldsmith, P. F. 2001, ApJ, 557, 736

Gómez, L., Wyrowski, F., Pillai, T., Leurini, S., \& Menten, K. M. 2011, A\&A, 529, A161

Griffin, M. J., Abergel, A., Abreu, A., et al. 2010, A\&A, 518, L3

Gritschneder, M., Lin, D. N. C., Murray, S. D., Yin, Q.-Z., \& Gong, M.-N. 2012, ApJ, 745, 22

Hatchell, J., \& van der Tak, F. F. S. 2003, A\&A, 409, 589

Heitsch, F., \& Hartmann, L. 2008, ApJ, 689, 290

Hennemann, M., Birkmann, S. M., Krause, O., \& Lemke, D. 2008, A\&A, 485, 753

Hennemann, M., Motte, F., Schneider, N., et al. 2012, A\&A, 543, L3

Hildebrand, R. H. 1983, QJRAS, 24, 267

Hill, T., Motte, F., Didelon, P., et al. 2011, A\&A, 533, A94

Johnstone, D., Fiege, J. D., Redman, R. O., Feldman, P. A., \& Carey, S. J. 2003, ApJ, 588, L37

Jørgensen, J. K., Schöier, F. L., \& van Dishoeck, E. F. 2004, A\&A, 416, 603

Kessler, M. F., Steinz, J. A., Anderegg, M. E., et al. 1996, A\&A, 315, L27

Klessen, R. S., Ballesteros-Paredes, J., Vázquez-Semadeni, E., \& Durán-Rojas, C. 2005, ApJ, 620, 786

Ladd, N., Purcell, C., Wong, T., \& Robertson, S. 2005, PASA, 22, 62

Launhardt, R., Stutz, A. M., Schmiedeke, A., et al. 2013, A\&A, 551, A98

López-Sepulcre, A., Walmsley, C. M., Cesaroni, R., et al. 2011, A\&A, 526, L2

Mac Low, M.-M., \& Klessen, R. S. 2004, Rev. Mod. Phys., 76, 125

MacLaren, I., Richardson, K. M., \& Wolfendale, A. W. 1988, ApJ, 333, 821

McKee, C. F., \& Tan, J. C. 2003, ApJ, 585, 850

Men'shchikov, A., André, P., Didelon, P., et al. 2010, A\&A, 518, L103

Molinari, S., Swinyard, B., Bally, J., et al. 2010, PASP, 122, 314

Motte, F., Schilke, P., \& Lis, D. C. 2003, ApJ, 582, 277

Müller, H. S. P., Schlöder, F., Stutzki, J., \& Winnewisser, G. 2005, J. Mol. Struct., 742,215

Nguyen Luong, Q., Motte, F., Schuller, F., et al. 2011, A\&A, 529, A41

Ossenkopf, V., \& Henning, T. 1994, A\&A, 291, 943

Ott, S. 2010, in Astronomical Data Analysis Software and Systems XIX, eds.

Y. Mizumoto, K.-I. Morita, \& M. Ohishi, ASP Conf. Ser., 434, 139

Perault, M., Omont, A., Simon, G., et al. 1996, A\&A, 315, L165 
J. Tackenberg et al.: Flows along massive star-forming regions

Peretto, N., \& Fuller, G. A. 2009, A\&A, 505, 405

Peretto, N., \& Fuller, G. A. 2010, ApJ, 723, 555

Peretto, N., André, P., \& Belloche, A. 2006, A\&A, 445, 979

Peretto, N., André, P., Könyves, V., et al. 2012, A\&A, 541, A63

Peters, T., Banerjee, R., Klessen, R. S., et al. 2010, ApJ, 711, 1017

Pilbratt, G. L., Riedinger, J. R., Passvogel, T., et al. 2010, A\&A, 518, L1

Pillai, T., Wyrowski, F., Carey, S. J., \& Menten, K. M. 2006, A\&A, 450, 569

Poglitsch, A., Waelkens, C., Geis, N., et al. 2010, A\&A, 518, L2

Ragan, S., Henning, T., Krause, O., et al. 2012a, A\&A, 547, A49

Ragan, S. E., Heitsch, F., Bergin, E. A., \& Wilner, D. 2012b, ApJ, 746, 174

Roussel, H. 2013, PASP, 125, 1126

Sakai, T., Sakai, N., Hirota, T., \& Yamamoto, S. 2010, ApJ, 714, 1658

Sawada, T., Ikeda, N., Sunada, K., et al. 2008, PASJ, 60, 445

Schlingman, W. M., Shirley, Y. L., Schenk, D. E., et al. 2011, ApJS, 195, 14

Schneider, N., Csengeri, T., Bontemps, S., et al. 2010, A\&A, 520, A49

Schöier, F. L., van der Tak, F. F. S., van Dishoeck, E. F., \& Black, J. H. 2005,

A\&A, 432, 369

Schuller, F., Menten, K. M., Contreras, Y., et al. 2009, A\&A, 504, 415

Smith, R. J., Longmore, S., \& Bonnell, I. 2009, MNRAS, 400, 1775

Smith, R. J., Shetty, R., Beuther, H., Klessen, R. S., \& Bonnell, I. A. 2013, ApJ, 771,24
Sridharan, T. K., Beuther, H., Schilke, P., Menten, K. M., \& Wyrowski, F. 2002, ApJ, 566, 931

Sridharan, T. K., Beuther, H., Saito, M., Wyrowski, F., \& Schilke, P. 2005, ApJ, 634, L57

Sunada, K., Yamaguchi, C., Kuno, N., et al. 2000, in Imaging at Radio through Submillimeter Wavelengths, eds. J. G. Mangum, \& S. J. E. Radford, ASP Conf. Ser., 217, 19

Tackenberg, J., Beuther, H., Henning, T., et al. 2014, A\&A, submitted

Tielens, A. G. G. M. 2005, The Physics and Chemistry of the Interstellar Medium (Cambridge: Cambridge University Press)

Tobin, J. J., Hartmann, L., Bergin, E., et al. 2012, ApJ, 748, 16

Vasyunina, T., Linz, H., Henning, T., et al. 2009, A\&A, 499, 149

Vasyunina, T., Linz, H., Henning, T., et al. 2011, A\&A, 527, A88

Wang, Y., Zhang, Q., Pillai, T., Wyrowski, F., \& Wu, Y. 2008, ApJ, 672, L33

Wienen, M., Wyrowski, F., Schuller, F., et al. 2012, A\&A, 544, A146

Wilcock, L. A., Kirk, J. M., Stamatellos, D., et al. 2011, A\&A, 526, A159

Wilcock, L. A., Ward-Thompson, D., Kirk, J. M., et al. 2012, MNRAS, 422, 1071

Williams, J. P., de Geus, E. J., \& Blitz, L. 1994, ApJ, 428, 693

Zinnecker, H., \& Yorke, H. W. 2007, ARA\&A, 45, 481

Pages 22 to 25 are available in the electronic edition of the journal at http://www . aanda. org 
Table 3. $870 \mu \mathrm{m}$ ATLASGAL clump properties.

\begin{tabular}{|c|c|c|c|c|c|c|c|c|c|c|c|}
\hline IRDC ident & RA & Dec & {$\left[10^{22} \mathrm{~cm}^{-2}\right]$} & {$\left[M_{\odot}\right]$} & $R_{\mathrm{eff}}$ & $\begin{array}{l}\mathrm{N}_{2} \mathrm{H}^{+} \\
\text {abun- } \\
\text { dance } \\
{\left[10^{12}\right]}\end{array}$ & {$\left[\mathrm{km} \mathrm{s}^{-1}\right]$} & $\Delta v_{\mathrm{N}_{2} \mathrm{H}^{+}}$ & $\begin{array}{l}\text { aver. } \\
\Delta v_{\mathrm{N}_{2} \mathrm{H}^{+}} \\
{\left[\mathrm{km} \mathrm{s}^{-1}\right]}\end{array}$ & $M_{\mathrm{vir}, k}=128$ & $M_{\mathrm{vir}, k=190}$ \\
\hline 18102 & 181311.2 & -1180006 & 11.2 & 1019. & 57.1 & 3.0 & 21.7 & 3.2 & 2.7 & 680 & 1026 \\
\hline $18151-11^{1}$ & 181758.2 & -1120726 & 32.2 & 677. & 34.5 & 1.1 & 33.0 & 1.9 & 2.0 & 232 & 350 \\
\hline $18151-12^{1}$ & 181750.3 & -1120754 & 20.4 & 524. & 32.5 & 1.3 & 29.7 & 3.1 & 3.1 & 495 & 747 \\
\hline $18151-13^{1}$ & 181751.9 & -1120654 & 6.6 & 184. & 23.7 & $-1-1-1$ & $-1-1-1$ & $-1-1-1$ & 0.7 & 19 & 29 \\
\hline $18151-14$ & 181755.7 & -1120654 & 2.6 & 21. & 9.0 & $-1-1-1$ & $-1-1-1$ & $-1-1-1$ & 3.0 & 128 & 193 \\
\hline $18182-11^{1}$ & 182109.2 & -1143147 & 17.2 & 1032. & 36.3 & 1.4 & 59.7 & 2.7 & 2.6 & 515 & 777 \\
\hline $18182-12^{7}$ & 182114.8 & -1143304 & 2.9 & 382. & 33.7 & 5.5 & 41.0 & 1.6 & 1.9 & 256 & 387 \\
\hline $18182-14^{7}$ & 182113.8 & -1143411 & 1.6 & 100. & 20.3 & 3.3 & 41.2 & 1.9 & 2.3 & 217 & 327 \\
\hline $18223-11^{1}$ & 182510.6 & -1124224 & 8.1 & 975. & 43.1 & 4.7 & 44.2 & 1.9 & 2.0 & 358 & 53 \\
\hline $18223-13^{1}$ & 182508.3 & -1124523 & 4.9 & 449. & 32.6 & 9.2 & 44.6 & 2.9 & 2.9 & 599 & 90 \\
\hline $18223-12^{1}$ & 182509.5 & -1124411 & 3.3 & 374. & 31.6 & 10.1 & 44.6 & 2.5 & 2.3 & 361 & 54 \\
\hline 18306-11 & 183323.6 & -1083336 & 9.0 & 640. & 32.3 & 4.8 & 78.0 & 2.7 & 2.7 & 536 & 809 \\
\hline $18306-12$ & 183331.6 & -1083236 & 2.0 & 107. & 18.8 & $-1-1-1$ & $-1-1-1$ & $-1-1-1$ & 1.6 & 104 & 158 \\
\hline $18308-11^{1}$ & 183332.6 & -1083916 & 8.2 & 1483. & 40.3 & 3.9 & 76.6 & 3.0 & 3.4 & 1271 & 1916 \\
\hline $18308-13^{1}$ & 183329.8 & -1083827 & 2.7 & 592. & 33.7 & 6.5 & 74.7 & 2.7 & 3.0 & 796 & 1201 \\
\hline $18308-14^{1}$ & 183327.0 & -1083853 & 1.6 & 81. & 14.0 & $-1-1-1$ & $-1-1-1$ & $-1-1-1$ & 0.9 & 33 & 50 \\
\hline $18308-15$ & 183334.6 & -1083555 & 1.4 & 61. & 12.7 & 10.6 & 77.8 & 1.8 & 1.6 & 83 & 125 \\
\hline $18308-16$ & 183336.2 & -1083641 & 1.4 & 57. & 12.2 & $-1-1-1$ & $-1-1-1$ & $-1-1-1$ & 1.2 & 48 & 72 \\
\hline $18310-11^{1}$ & 183347.7 & -1082351 & 6.5 & 1100. & 33.2 & 9.0 & 83.2 & 1.9 & 2.4 & 566 & 854 \\
\hline $18310-12^{1}$ & 183343.7 & -1082125 & 6.3 & 1236. & 33.5 & 6.0 & 84.3 & 3.7 & 3.5 & 1209 & 1824 \\
\hline $18310-14^{1}$ & 183339.2 & -1082111 & 2.6 & 346. & 23.2 & 9.4 & 86.1 & 2.4 & 2.3 & 384 & 579 \\
\hline $18310-13^{1}$ & 183343.8 & -1082213 & 1.5 & 221. & 21.1 & $-1-1-1$ & $-1-1-1$ & $-1-1-1$ & 3.1 & 612 & 924 \\
\hline $18337-14$ & 183637.0 & -1073853 & 2.1 & 214. & 26.0 & $-1-1-1$ & $-1-1-1$ & $-1-1-1$ & 2.9 & 497 & 749 \\
\hline $18337-13^{1}$ & 183618.2 & -1074106 & 1.9 & 177. & 24.9 & 5.4 & 56.0 & 2.6 & 2.6 & 371 & 560 \\
\hline $18337-15$ & 183618.7 & -1074143 & 1.7 & 137. & 22.4 & $-1-1-1$ & $-1-1-1$ & $-1-1-1$ & 2.8 & 394 & 594 \\
\hline $18337-12^{1}$ & 183626.5 & -1074115 & 1.5 & 44. & 12.7 & 2.9 & 57.4 & 1.1 & 1.1 & 36 & 55 \\
\hline $\begin{array}{l}18454- \\
\mathrm{mm}^{4}\end{array}$ & 184746.7 & -1015426 & 80.8 & 16117. & 50.6 & 2.6 & 95.6 & 5.4 & 6.3 & 6552 & 9880 \\
\hline $18454-10$ & 184749.4 & -1015359 & 8.4 & 3032. & 40.1 & 12.5 & 96.6 & 7.3 & 6.0 & 4787 & 7219 \\
\hline $18454-11$ & 184738.9 & -1015655 & 8.0 & 2641. & 38.2 & - & - & - & - & - & - \\
\hline $18454-3^{2}$ & 184755.4 & -1015331 & 6.4 & 2089. & 41.2 & 8.9 & 95.3 & 6.0 & 6.8 & 6161 & 9290 \\
\hline $18454-12$ & 184742.2 & -1015612 & 5.3 & 1358. & 35.3 & - & - & - & 3.9 & 1729 & 2608 \\
\hline 18454-13 & 184740.4 & -1015559 & 5.3 & 1704. & 29.9 & - & - & - & 3.5 & 1204 & 1816 \\
\hline $18454-14$ & 184748.2 & -1015753 & 4.0 & 1095. & 36.8 & 6.3 & 92.8 & 2.7 & 3.1 & 1165 & 1757 \\
\hline $18454-15$ & 184749.1 & -1015739 & 3.6 & 782. & 27.7 & 7.5 & 93.9 & 3.2 & 3.3 & 994 & 1499 \\
\hline $18454-1^{2}$ & 184802.0 & -1015401 & 3.4 & 608. & 25.6 & 6.9 & 99.3 & 2.6 & 2.7 & 613 & 925 \\
\hline $18454-4^{2}$ & 184801.1 & -1015227 & 3.3 & 443. & 22.4 & 6.3 & 100.3 & 1.8 & 1.6 & 179 & 270 \\
\hline $18454-2^{2}$ & 184759.8 & -1015412 & 3.3 & 666. & 27.5 & - & - & - & 3.2 & 913 & 1377 \\
\hline 18454-16 & 184751.3 & -1015306 & 2.5 & 354. & 20.6 & - & - & - & 8.3 & 4660 & 7028 \\
\hline $18454-5 b^{2}$ & 184757.7 & -1015618 & 2.4 & 261. & 18.2 & 7.5 & 93.8 & 2.6 & 2.7 & 428 & 646 \\
\hline $18454-7^{2}$ & 184752.3 & -1015502 & 2.4 & 190. & 15.9 & 10.4 & 94.2 & 2.6 & 2.7 & 373 & 563 \\
\hline $18454-17$ & 184752.5 & -1015309 & 2.1 & 240. & 18.5 & - & - & - & 9.3 & 5237 & 7897 \\
\hline $18454-6^{2}$ & 184802.1 & -1015543 & 1.9 & 115. & 13.1 & - & - & - & - & - & - \\
\hline 18454-19 & 184751.6 & -1015825 & 1.8 & 171. & 16.6 & - & - & - & 2.7 & 399 & 603 \\
\hline $18454-9^{2}$ & 184757.3 & -1015735 & 1.6 & 199. & 18.2 & - & - & - & 2.6 & 395 & 596 \\
\hline $18454-21$ & 184748.5 & -1015520 & 1.6 & 212. & 18.8 & - & - & - & 4.1 & 1048 & 1581 \\
\hline 1930-1 & 182558.4 & -1120401 & 4.0 & 164. & 30.1 & 10.8 & 26.6 & 2.7 & 2.6 & 299 & 451 \\
\hline $1930-2$ & 182552.3 & -1120456 & 2.9 & 93. & 23.9 & 7.9 & 27.0 & 2.2 & 2.2 & 174 & 263 \\
\hline $1930-3$ & 182553.8 & -1120440 & 2.0 & 94. & 26.2 & 8.9 & 26.7 & 2.1 & 2.1 & 171 & 257 \\
\hline G11.11-1 ${ }^{3}$ & 181028.1 & -1192235 & 5.5 & 455. & 30.1 & 5.3 & 29.5 & 1.9 & 1.9 & 234 & 352 \\
\hline G11.11-6 $6^{3}$ & 181007.0 & -1192906 & 4.3 & 411. & 31.4 & 4.7 & 29.8 & 1.7 & 1.9 & 237 & 358 \\
\hline G11.11- $7^{3}$ & 181006.5 & -1192740 & 3.9 & 458. & 31.0 & 2.9 & 29.4 & 2.3 & 2.4 & 363 & 547 \\
\hline G11.11-2 3 & 181033.3 & -1192200 & 3.2 & 325. & 29.3 & 8.0 & 30.5 & 1.9 & 2.0 & 249 & 376 \\
\hline G11.11-9 & 181032.9 & -1192211 & 3.0 & 83. & 14.0 & 8.0 & 30.5 & 1.9 & 1.9 & 104 & 157 \\
\hline G11.11-10 & 181005.2 & -1192634 & 2.8 & 109. & 18.2 & - & - & - & 2.2 & 178 & 268 \\
\hline G11.11-11 & 181005.7 & -1192646 & 2.7 & 130. & 19.1 & - & - & - & 2.2 & 198 & 299 \\
\hline G11.11-12 & 181018.0 & -1192436 & 2.6 & 199. & 25.1 & 7.8 & 29.9 & 1.7 & 1.7 & 158 & 238 \\
\hline
\end{tabular}

Notes. The columns are as follows: clump identifier, as displayed in the figues; names are adopted from ${ }^{1}$ Beuther et al. $(2002 \mathrm{~b}) ;{ }^{7}$ Beuther et al. (2007); ${ }^{2}$ Beuther et al. (2012); ${ }^{3}$ Johnstone et al. (2003); ${ }^{4}$ Motte et al. (2003); ${ }^{5}$ Vasyunina et al. (2009); and ${ }^{6}$ Wang et al. (2008). 
Table 3. continued.

\begin{tabular}{|c|c|c|c|c|c|c|c|c|c|c|c|}
\hline IRDC ident & RA & Dec & $\begin{array}{l}N_{\mathrm{H}_{2}} \\
{\left[10^{22} \mathrm{~cm}^{-2}\right]}\end{array}$ & {$\left[M_{\odot}\right]$} & {$\left[{ }^{\prime \prime}\right]$} & {$\left[10^{12}\right]$} & {$\left[\mathrm{km} \mathrm{s}^{-1}\right]$} & {$\left[\mathrm{km} \mathrm{s}^{-1}\right]$} & $\begin{array}{l}\text { aver. } \\
\Delta v_{\mathrm{N}_{2} \mathrm{H}^{+}} \\
{\left[\mathrm{km} \mathrm{s}^{-1}\right]}\end{array}$ & $\begin{array}{l}M_{\mathrm{vir}, k=128} \\
{\left[M_{\odot}\right]}\end{array}$ & $\begin{array}{l}M_{\mathrm{vir}, k}=190 \\
{\left[M_{\odot}\right]}\end{array}$ \\
\hline G11.11-13 & 181032.3 & -1192227 & 2.5 & 237. & 26.9 & 7.9 & 30.4 & 1.8 & 1.9 & 194 & 292 \\
\hline G11.11-14 & 181005.4 & -1192718 & 2.5 & 134. & 19.1 & - & - & - & 2.3 & 205 & 309 \\
\hline G11.11-15 & 181025.7 & -1192258 & 2.5 & 166. & 22.2 & 10.7 & 29.2 & 1.0 & 1.3 & 74 & 112 \\
\hline G11.11-16 & 181004.8 & -1192733 & 2.5 & 109. & 18.5 & - & - & - & 2.0 & 158 & 238 \\
\hline G11.11-17 & 181013.9 & -1192417 & 2.1 & 106. & 19.7 & 3.9 & 30.3 & 1.4 & 1.2 & 59 & 89 \\
\hline G11.11-18 & 181021.4 & -1192347 & 1.8 & 70. & 16.3 & 8.5 & 29.3 & 1.6 & 1.6 & 86 & 130 \\
\hline G11.11-19 & 181034.2 & -1192059 & 1.7 & 73. & 16.9 & 17.4 & 31.1 & 1.7 & 1.5 & 82 & 124 \\
\hline G11.11-20 & 181022.0 & -1192331 & 1.7 & 100. & 20.3 & 8.2 & 29.2 & 1.6 & 1.6 & 110 & 166 \\
\hline G11.11-21 & 181005.8 & -1192605 & 1.6 & 75. & 17.9 & 7.7 & 28.6 & 1.9 & 1.7 & 112 & 170 \\
\hline G11.11-22 & 181021.0 & -1192410 & 1.6 & 72. & 17.3 & 10.7 & 29.4 & 1.6 & 1.7 & 102 & 154 \\
\hline G11.11-23 & 181015.5 & -1192437 & 1.5 & 37. & 12.2 & 7.4 & 30.2 & 1.5 & 0.9 & 19 & 30 \\
\hline G13-4 & 181734.9 & -1170643 & 4.0 & 370. & 42.4 & 4.7 & 21.7 & 2.0 & 1.5 & 155 & 235 \\
\hline G13-3 & 181721.5 & -1170918 & 2.0 & 70. & 21.4 & 6.6 & 23.3 & 1.4 & 1.8 & 112 & 169 \\
\hline G13-1 & 181719.2 & -1170941 & 1.6 & 54. & 19.7 & - & - & - & 3.5 & 379 & 571 \\
\hline G13-2 & 181734.0 & -1170527 & 1.5 & 20. & 12.2 & - & - & - & 1.6 & 52 & 78 \\
\hline G1505 & 181739.0 & -1154853 & 1.4 & 36. & 14.8 & 11.1 & 29.9 & 1.3 & 1.3 & 47 & 71 \\
\hline$G 2823-2^{6}$ & 184251.9 & -1035952 & 15.9 & 3136. & 51.1 & 3.1 & 78.1 & 3.4 & 3.3 & 1509 & 2276 \\
\hline G2823-3 & 184237.3 & -1040209 & 5.7 & 890. & 35.2 & 3.6 & 80.8 & 2.5 & 2.5 & 626 & 944 \\
\hline $\mathrm{G} 2823-1^{6}$ & 184250.4 & -1040315 & 4.6 & 919. & 37.4 & 7.7 & 79.2 & 3.4 & 3.5 & 1234 & 1861 \\
\hline G2823-4 & 184248.9 & -1040224 & 3.5 & 502. & 28.9 & 8.1 & 80.0 & 2.7 & 3.0 & 722 & 1089 \\
\hline G2823-5 & 184253.6 & -1040233 & 3.0 & 771. & 36.3 & 8.8 & 79.2 & 2.6 & 2.9 & 860 & 1298 \\
\hline G2823-6 & 184246.5 & -1040414 & 2.8 & 658. & 34.2 & 5.4 & 79.3 & 3.0 & 3.1 & 883 & 1331 \\
\hline G2823-7 & 184253.3 & -1040057 & 1.9 & 118. & 16.3 & 13.5 & 79.3 & 2.2 & 2.3 & 247 & 372 \\
\hline G2823-8 & 184242.7 & -1040143 & 1.8 & 176. & 20.0 & 5.1 & 80.8 & 2.6 & 2.6 & 362 & 546 \\
\hline G2823-9 & 184239.7 & -1040033 & 1.8 & 85. & 14.0 & 13.5 & 81.4 & 2.2 & 2.2 & 180 & 272 \\
\hline G2823-10 & 184254.7 & -1040108 & 1.7 & 145. & 18.2 & 11.6 & 79.3 & 2.1 & 2.2 & 240 & 362 \\
\hline
\end{tabular}




\section{Appendix A}

\section{A.1. Description of the linewidth distribution}

IRDC 18223: we found a clear increase of the linewidth toward the center of IRDC 18223-2, but IRDC 18223-2 and -3 show an increase toward the edge.

IRDC 18310: in IRDC 18310-1 the linewidth increases not toward the submm peak, but toward the detected PACS point sources within. IRDC $18310-4$ is a $70 \mu \mathrm{m}$ dark region and the linewidth increases toward its edge. In between IRDC 18310-2, -3 , and -4 the measured increase in linewidth is due to two independent, overlapping components.

G11.11: while for the two main peaks of G11.11, G11.11-1, and G11.11-2, the linewidth increases prototypically toward its edges, the other clumps of the northern part shown in Fig. 2 show no such clear variation of the linewidth. The systematic offset in the mapping of the southern part of G11.11 does not allow a systematic study of the line parameters. Nevertheless, it is worth noting that the linewidth of the southern part is significantly broader than it is for the northern mapped part.

G15.05: no clumps detected at given column density threshold. IRDC 18102: we found an increase in linewidth toward the submm peak with its connected PACS point source. An additional broadening of the linewidth from east to south is prominent as well.

IRDC 18151: in IRDC 18151, IRDC 18151-2 has the widest linewidth with $3.1 \mathrm{~km} \mathrm{~s}^{-1}$. However, it is known to have an outflow (Beuther \& Sridharan 2007), which migh explain the broad linewidth. Both brighter at PACS $70 \mu \mathrm{m}$ (the associated PACS point source is with $4026 L_{\odot} \sim 10$ times more luminous than the one connected to IRDC 18151-2) and with a collimated outflow (Fallscheer et al. 2011) as well, IRDC 18151-1 has a linewidth of only $1.9 \mathrm{~km} \mathrm{~s}^{-1}$. Along the dust continuum emission away from the center the linewidth increases again. However, the reported outflow is perpendicular to the dust continuum emission and therefore cannot explain the broadening of the linewidth. Despite its still luminous PACS point source of $184 L_{\odot}$, IRDC $18151-3$ has the narrowest linewidth in this region with $0.7 \mathrm{~km} \mathrm{~s}^{-1}$. That is consistent with the fact that López-Sepulcre et al. (2011) found no outflows toward that source.

IRDC 18182: the well-studied HMPO in the north with four outflows connected to it (Beuther et al. 2006) has a broadening linewidth toward its center. The region of interest, the
IRDC in the southeast has its narrowest linewidth at the peak of IRDC 18182-2. Although there is a PACS continuum source at its peak, Beuther \& Sridharan (2007) detected no SiO toward that peak. The southern part of the IRDC, IRDC 18182-4 has a slightly broader linewidth.

IRDC 18308: while the IR dark filament, connected to IRDC 18308-5 and IRDC 18308-6, has a similar linewidth all along between $1 \mathrm{~km} \mathrm{~s}^{-1}$ and $2 \mathrm{~km} \mathrm{~s}^{-1}$, the southern complex of IRDC 18308 has linewidths broader than $2.5 \mathrm{~km} \mathrm{~s}^{-1}$. However, the peak of the linewidth is offset from the submm peaks, but is north of IRDC 18308-1. It is interesting to note that the $\mathrm{N}_{2} \mathrm{H}^{+}$ column density is not aligned with the submm continuum peak IRDC 18308-1, and that the $\mathrm{N}_{2} \mathrm{H}^{+}$linewidth peak is offset from the $\mathrm{N}_{2} \mathrm{H}^{+}$column density peak. While the offset between the continuum and the $\mathrm{N}_{2} \mathrm{H}^{+}$emission peak of $\sim 15^{\prime \prime}$ might be explained by the pointing uncertainties, the offset between the column density peak and the linewidth peak of $\sim 42^{\prime \prime}$ are not. Since the linewidth and $\mathrm{N}_{2} \mathrm{H}^{+}$column density are measured from the same data, positional uncertainties are not an explanation for their difference of $30^{\prime \prime}$. As described in 3.3, we checked for the peak of the linewidth map whether its spectrum is fit better by two independent $\mathrm{N}_{2} \mathrm{H}^{+}$velocity components, but only founnd a single component. Because of the high-velocity resolution of $0.1 \mathrm{~km} \mathrm{~s}^{-1}$ for that check we can exclude an additional component as the cause of this broadening.

G19.30: the linewidth distribution in G19.30 peaks toward the two more massive submm peaks G19.30-1 and -2 with an additional increase in linewidth north of G19.30-1. In between P1 and P2, the linewidth is narrowest within G19.30-3, but still broad with $\sim 2 \mathrm{~km} \mathrm{~s}^{-1}$.

G28.34: the linewidth in G28.34 is in general very broad with $\Delta v>2 \mathrm{~km} \mathrm{~s}^{-1}$ with only few exceptions. It peaks toward the two main peaks G28.34-1 and -2 with linewidths of up to $3.5 \mathrm{~km} \mathrm{~s}^{-1}$. The narrowest linewidth is found to be in between the two main peaks at G28.34-10 at a linewidth of $2.1 \mathrm{~km} \mathrm{~s}^{-1}$. Using the maps smoothed to a velocity resolution of $0.4 \mathrm{~km} \mathrm{~s}^{-1}$, we found even narrower linewidths along the IR-dark filament beyond G28.34-9.

G48.66: the linewidths in G48 are narrower than those of other regions mapped within this sample. For the part where $\mathrm{N}_{2} \mathrm{H}^{+}$is detected, the region with the highest absorption and the embedded but detected PACS sources, the velocity dispersion is broadest and decreases along the filament to lower column densities. 
A.2.1. Nobeyama $45 \mathrm{~m}$ data

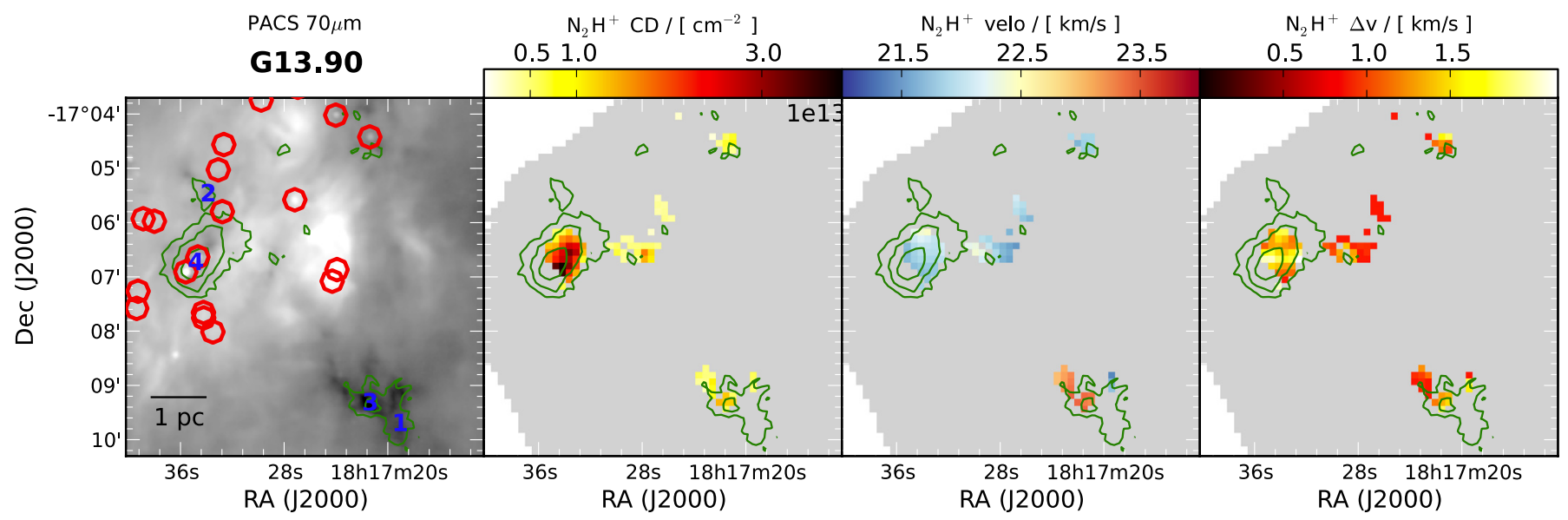

Fig. A.1. Parameter map of the omitted region G13.90 mapped with the Nobeyama $45 \mathrm{~m}$ telescope. The left panel shows the PACS $70 \mu \mathrm{m}$ maps with the PACS point sources detected by Ragan et al. (2012a) indicated by red circles, the blue numbers refer to the submm continuum peaks as given in Table 3. The second panel displays the $\mathrm{N}_{2} \mathrm{H}^{+}$column density derived from fitting the full $\mathrm{N}_{2} \mathrm{H}^{+}$hyperfine structure. The third and fourth panels show the corresponding velocity and linewidth (FWHM) of each fit. The contours from ATLASGAL $870 \mu \mathrm{m}$ are plotted with the lowest level representing $0.31 \mathrm{Jy}$, and continue in steps of $0.3 \mathrm{Jy}$.

\section{A.2.2. MOPRA data}

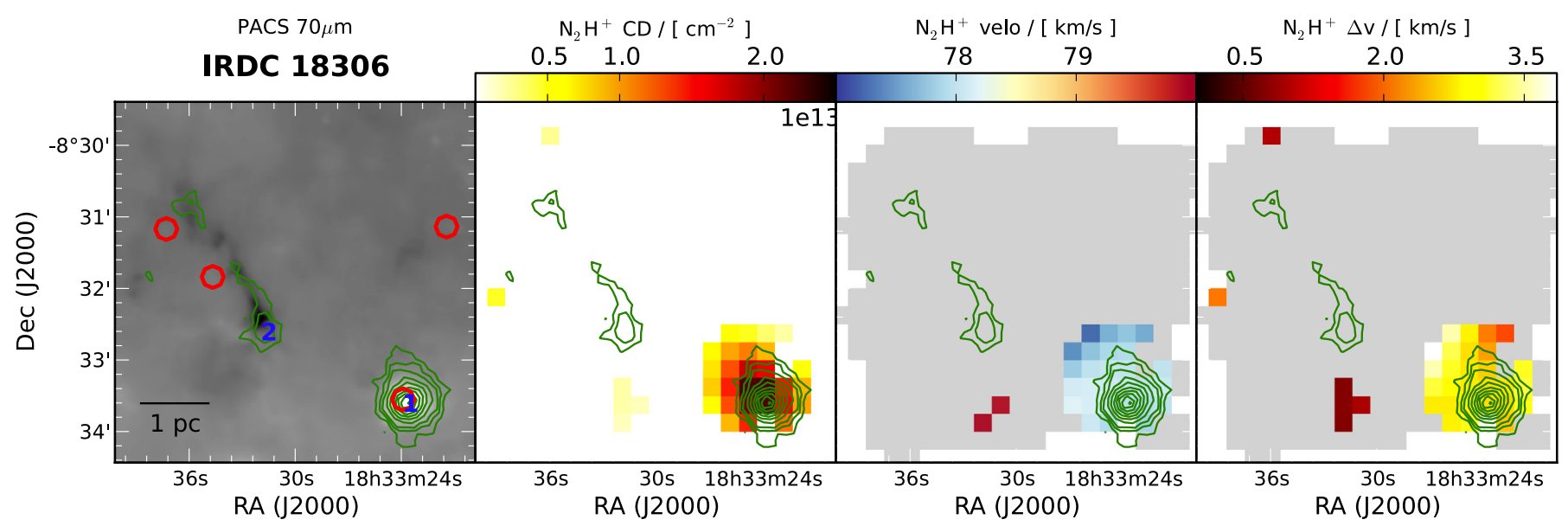

Fig. A.2. Parameter maps of the region IRDC 18306, mapped with the MOPRA telescope. The left panel shows the PACS $70 \mu \mathrm{m}$ maps with the PACS point sources detected by Ragan et al. (2012a) indicated by red circles, the blue numbers refer to the submm continuum peaks as given in Table 3. The second panel displays the $\mathrm{N}_{2} \mathrm{H}^{+}$column density derived from fitting the full $\mathrm{N}_{2} \mathrm{H}^{+}$hyperfine structure. The third and fourth panels show the corresponding velocity and linewidth (FWHM) of each fit. The green contours are from ATLASGAL $870 \mu \mathrm{m}$ at $0.31 \mathrm{Jy}, 0.46 \mathrm{Jy}$, and $0.61 \mathrm{Jy}$, continuing in steps of $0.3 \mathrm{Jy}$. The velocity resolution is smoothed to $0.4 \mathrm{~km} \mathrm{~s}^{-1}$ to improve the signal-to-noise ratio and increase the number of detected $\mathrm{N}_{2} \mathrm{H}^{+}$positions.

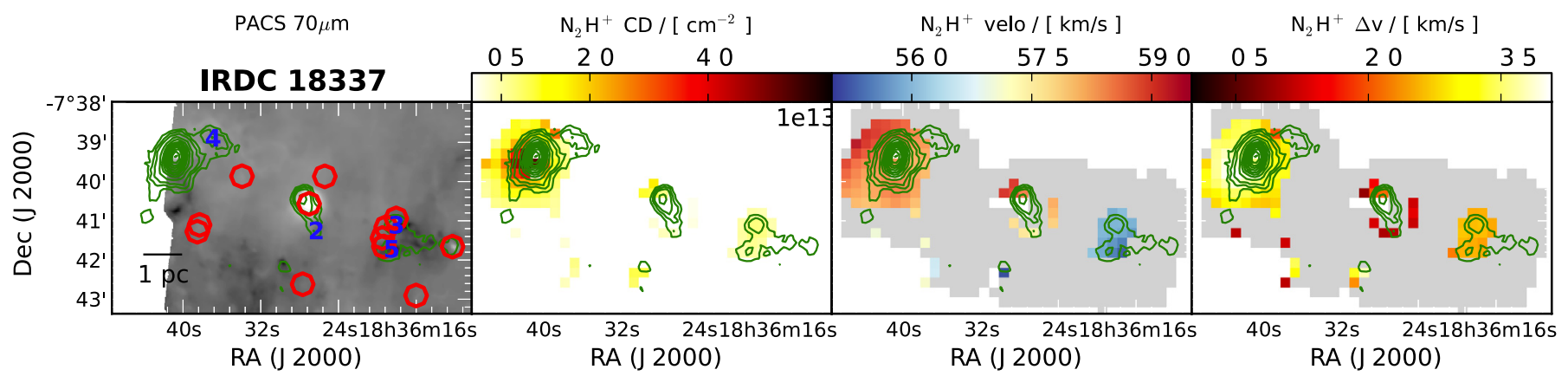

Fig. A.3. Same as Fig. A.2, but for the region IRDC 18337. 